Revue d'histoire de l'Amérique française

TALVUE D.HISTOIRE DE L'AMÉRIQUE FRANÇAISE

\title{
Sur quelques discontinuités dans l'expérience socio-économique du Québec : une hypothèse
}

\section{Gilles Paquet et Jean-Pierre Wallot}

Volume 35, numéro 4, mars 1982

URI : https://id.erudit.org/iderudit/304009ar

DOI : https://doi.org/10.7202/304009ar

Aller au sommaire du numéro

Éditeur(s)

Institut d'histoire de l'Amérique française

ISSN

0035-2357 (imprimé)

1492-1383 (numérique)

Découvrir la revue

Citer cet article

Paquet, G. \& Wallot, J.-P. (1982). Sur quelques discontinuités dans l'expérience socio-économique du Québec : une hypothèse. Revue d'histoire de l'Amérique française, 35(4), 483-521. https://doi.org/10.7202/304009ar d'utilisation que vous pouvez consulter en ligne. 


\title{
SUR QUELQUES DISCONTINUITÉS DANS L'EXPÉRIENCE SOCIO-ÉCONOMIQUE DU QUÉBEC: UNE HYPOTHÈSE*
}

\author{
GILLES PAQUET \\ Faculté d'administration \\ Université d'Ottawa \\ JEAN-PIERRE WALLOT \\ Département d'histoire \\ Université de Montréal
}

\begin{abstract}
«...starting from discontinuity... allows us to move from one question to another, steadily widening and deepening, discovering and creating the nature of our explicandum and delimiting it at the same time.» (Alexander Gerschenkron)
\end{abstract}

«...to map out the problem area and thus prepare the ground for its empirical investigation by appropriate methods.” (S. F. Nadel)

\section{Introduction}

Nos travaux des dernières années ont souligné l'importance de la mutation qui a modernisé le Québec au tournant du XIXe siècle. Les analyses des différentes arêtes de cette discontinuité nous ont amenés à mieux discerner la valeur heuristique d'une approche par les discontinuités et à l'exploiter dans une perspective séculaire pour l'étude de la dynamique de cette socio-économie. Car la césure du tournant du XIXe siècle ne représente que l'un des moments de transition entre régimes de fonctionnement dans cette évolution. D'où la recherche d'un découpage de ce temps long de l'expérience socio-économique du Québec en un certain nombre de tronçons correspondant à des régimes de fonctionnement caracté-

\footnotetext{
*Ce travail s'inscrit dans le cadre d'une étude des grandes discontinuités dans l'évolution de la réalité socio-matérielle du centre du Canada depuis le XVIIe jusqu'au «second XXe siècle» (Perroux). Nous tenons à remercier le ministère de l'Éducation du Québec (programme FCAC) ainsi que nos universités présentes et passées qui, à divers degrés, ont participé au financement de nos travaux. Nous exprimons également notre gratitude à nos collègues Steven Langdon et Wayne Smith pour des discussions fort utiles sur certains aspects de notre hypothèse, ainsi qu'à Andrée Giguère pour son aide dans la mise en forme du test préliminaire que nous en avons fait. Comme d'habitude, Rita Girard-Wallot a participé de multiples façons aux diverses étapes de ce travail et nous l'en remercions.
} 
ristiques. Ces tronçons sont articulés à certains moments-clés dans les discontinuités qui traduisent une cassure, un changement de rythme et de régime de fonctionnement, une transformation des structures.

L'historiographie canadienne et québécoise aligne un certain nombre de «scénarios» plus ou moins accrédités qui s'enracinent dans des cadres conceptuels divers. Ces scénarios repèrent une ou plusieurs discontinuités importantes, voire déterminantes, et caractérisent différemment les «séquences» ou régimes distincts qu'elles démarquent. 1760, 1783, 1840, 1867: autant de moments présumés importants dans l'un ou l'autre des schémas interprétatifs repris et amplifiés par les manuels.

Le présent article suggère un découpage de rechange ancré dans une vision différente et explicitée du procès socioéconomique. Ce découpage, nous le proposons comme une hypothèse de travail pouvant permettre d'organiser le contenu du fichier documentaire, d'encadrer certains schémas interprétatifs plus restreints, de résoudre certains paradoxes et d'intégrer de façon préliminaire un matériau riche, mais encore mal ou incomplètement exploré.

La section 2 définit à gros traits la problématique qui nous a guidés dans le démontage de l'expérience socio-économique du Québec sur plus de trois siècles. La section 3 ébauche les contours des quatre grandes discontinuités et des cinq régimes de fonctionnement qui s'y articulent. Enfin, la section 4 illustre l'utilité de ce cadre de référence pour enrichir certaines interprétations admises et pour identifier des pistes de recherche, avant la conclusion en section 5 .

L'institutionnalisme en science économique a des racines lointaines. On pourrait revenir à Karl Menger, à la fin du XIXe siècle en Europe, ou à J.R. Commons, aux ÉtatsUnis, dans l'entre-deux guerres. Ce mode de pensée a influencé de façon importante tous les travaux de l'école française d'après la seconde guerre mondiale, ainsi qu'en témoigne un numéro spécial de la Revue économique de novembre 1954. Dans les années 1960, on voit resurgir l'institutionnalisme dans la science économique anglo-saxonne et il s'impose en histoire économique. À ce mouvement plus récent, baptisé de néo-institutionnalisme, sont associés les noms de Harold Demsetz, Douglass North, John Hicks et nombre d'autres auteurs. Pour un coup d'oeil sur l'éventail de travaux logeant à cette enseigne, voir $\mathrm{H}$. Demsetz, «Toward a Theory of Property Rights», American Economics Association Papers \& Proceedings, mai 1967; John Hicks, A Theory of Economic History, Oxford, 1969; L. Davis et D.C. North, «Institutional Change and American Economic Growth: A First Step Towards a Theory of Institutional Innovation", Journal of Economic History, 30, mars 1970; A.A. Alchian et H. Demsetz, "The Property Right Paradigm», Journal of Economic History, mars 1973. 


\section{Une problématique}

Depuis une dizaine d'années, nos travaux en histoire économique du Canada et du Québec s'inscrivent dans le courant néoinstitutionnel. Cette étiquette assez ambiguë ne renvoie ni à un modèle univoque ni à une méthodologie stylisée. Elle désigne plutôt une stratégie de recherche qui mise avant tout sur la dimension institutionnelle/informationnelle des phénomènes comme voie d'accès à une compréhension de la dérive des socio-économies ${ }^{1}$.

À partir d'une vision de la socio-économie canadienne comme «procès institué» à la Polanyi ${ }^{2}$, nous avons opté pour une approche à la connaissance historique via le monde 3 (à la Popper). C'est un pari dont nous avons exposé les avantages stratégiques en 1979 dans un article de la Revue 3 . Sans en reprendre ici l'exposé, rappelons qu'il s'agit de partir de la trame institutionnelle / organisationnelle comme du faisceau constitutif d'une constellation d'armistices liant faits de conscience et faits matériels, comme d'un lieu privilégié pour capter la dynamique du procès évolutif de la socioéconomie.

Il nous a semblé que le meilleur moyen d'analyser ce procès institué toujours en train de se métamorphoser et de s'instituer différemment, de lieu en lieu et de moment en moment, c'était de recourir à la «méso-analyse» ${ }^{4}$, c'est-à-dire à la décomposition de ce «grand jeu» en un certain nombre de «sous-jeux» séparables. À partir de la composition et de l'imbrication de ces sous-jeux, chacun avec ses règles propres mais changeantes, il est possible d'étudier l'évolution du grand jeu.

Pour identifier les sous-jeux fondamentaux séparables, nous nous sommes inspirés de J. Akerman qui, dès les années 1940, avait distingué certaines «forces motrices» du développement et tenté de reconstruire à l'aide de ces «sous-procès» une explication rationnelle du développement des économies occidentales dans une perspective séculaire ${ }^{5}$. Nous avons reformulé le découpage d'Akerman

2 K. Polanyi, «The Economy as Instituted Process», in K. Polanyi, C.M. Arensberg, H.W. Pearson, ed., Trade and Markets in the Early Empires (New York, 1957), $243-70$

G. Paquet et J.-P. Wallot, «Pour une méso-histoire du XIXe siècle canadien», RHAF, 33,3 (décembre 1979): section 2.

4 Cette méso-analyse part de l'idée de procès socio-économiques comme de jeux avec leurs acteurs, leurs règles, leurs frontières, leur rythme. C'est une idée empruntée à Michel Crozier («The Relationship between Micro and Macrosociology», Human Relations, 25,3 (juillet 1972): 239-51). Nous l'avons développée dans la section 5 de G. Paquet \& J.-P. Wallot, $o p$. cit. Plus récemment, l'idée a été poussée un cran plus loin par Andrew Schotter, The Economic Theory of Social Institutions (Cambridge, 1981). Schotter y développe une approche néo-institutionnelle à l'économie à partir de la notion de jeu. 1955).

J. Akerman, Ekonomisk Teori II, Lund, 1944 (traduction française, Paris, 
de façon à en distiller six sous-procès fondamentaux séparables: la démographie, la production et l'échange, la finance, l'écologie des groupes sociaux et de leurs motivations, l'État, la répartition des revenus et de la richesse ${ }^{6}$. Ces sous-procès se superposent, s'intègrent, se combinent pour composer des secteurs caractérisés par un même tonus et des périodes déterminées par un même régime de fonctionnement.

Cette conjonction des sous-procès, nous l'avons présentée ailleurs (même si l'analogie n'est pas entièrement satisfaisante) comme le résultat de la superposition d'autant de "transparents» ou d'acétates composant simultanément les complexités horizontales et verticales de l'expérience d'une socio-économie concrète. Cet instrument d'analyse permet en effet de repérer dans l'espace (horizontalement) et dans le temps (verticalement) des zones caractérisées par un certain tonus ou une certaine stabilité de fonctionnement. Le tableau, qui émerge du «mixage» ou de l'interaction des sous-procès, reproduit donc simultanément la «cartographie» et la "périodisation» de l'expérience d'une socio-économie. Nous avons aussi avancé l'hypothèse qu'une analyse à ce niveau pourrait fort bien révéler à la fois les sources et les causes de certains métaprocès (l'industrialisation et l'urbanisation par exemple), ceux-ci apparaissant comme le résultat de certaines formes de symbiose et d'intercréation des sous-procès ${ }^{7}$.

La composition de ces sous-procès ou sous-jeux en une dynamique du grand jeu, en une explication de la dynamique évolutive de la socio-économie comme procès institué, pose de formidables problèmes théoriques, et nous sommes encore loin de pouvoir compter sur une théorie des procès qui explicite de manière précise

\footnotetext{
Ces six sous-procès ou sous-jeux sont une condensation des huit forces motrices identifiées par Akerman. On pourrait écrire un livre sur chacun de ces sous-procès et, de fait, on en a écrit plusieurs. Ce qu'il est important de comprendre, c'est que ces sous-procès résultent d'une application au monde des organisations d'un procédé bien connu de décomposition utilisé par les spécialistes de recherche opérationnelle (F. Bessière, «The Concept of Separability and the Optimization of Economic Organization», European Economic Review (automne 1969): 74-91). Les six sous-procès retenus ont d'abord le mérite d'être facilement repérables et d'avoir des frontières, des règles de fonctionnement et une trame organisationnelle qu'on peut décrire et calibrer. Ils regroupent aussi les forces les plus importantes d'une société pour une reconstruction théorique: celles qu'on doit prendre en considération avant toute autre, selon Akerman. Enfin, ils ont l'avantage d'être intuitivement assez faciles à comprendre et de correspondre à un ensemble de travaux disciplinaires abondants menés par des historiens et des spécialistes d'autres sciences humaines depuis une ou deux générations au Canada et au Québec. Voilà qui permet d'emprunter à ces disciplines leurs discours et leurs résultats. Nous avons suggéré ailleurs (G. Paquet et J.-P. Wallot, op. cit., note 82) une liste partielle de ces travaux.

Certains éléments de cette approche sont esquissés dans G. Paquet et J.-P. Wallot, op. cit., section 5.c. Pour ce qui est de l'analyse des méta-procès, voir G. Paquet et J.-P. Wallot, "Canadian Cities as Social Technologies: An Exploratory Essay" in W. Borah, J. Hardoy, G.A. Stelter, ed., Urbanization in the Americas (Ottawa, 1980), 57-62 (un numéro spécial de la Revue d'histoire urbaine/Urban History Review).
} 
et entièrement satisfaisante les modes de composition et d'intercréation des sous-procès. Le programme de recherche dans ce domaine est déjà ancien et on en trouve prospectivement les différents stades bien définis déjà en 1954: mais les travaux n'ont guère progressé au-delà du premier débrouissaillement ${ }^{8}$.

Pourtant, il est clair qu'on ne peut s'arrêter aux simples patterns définis par la superposition plus ou moins mécanique des sous-procès. Il faut cerner plutôt les réseaux de relations qui sont les sources vives de l'intercréation entre sous-procès. Or, pour le moment, les études ne dépassent guère le niveau du pattern ${ }^{9}$. Une véritable analyse au niveau des réseaux tend pour sa part à débusquer (ou tout au moins cherche à le faire) une sorte de «rationalité de fonctionnement» qui confère au procès institué son sens et explique sa dérive ${ }^{10}$.

Comprendre la rationalité de fonctionnement d'un réseau, sa logique (et donc ce qui confère leurs particularités et leur logique à un sous-système et à son fonctionnement), implique pour nous la prise en considération de chaque sous-procès comme mécanisme concret élaboré par les hommes pour régler leur coopération,

8 Dans le numéro spécial de novembre 1954 de la Revue économique, J. L’Homme («Matériaux pour une théorie de la structure économique et sociale», surtout, 847-57) suggère une série d'opérations en quatre étapes: (1) découpage du système en parties, (2) examen des relations internes et externes, quantitatives et qualitatives qui les unissent, (3) repérage des relations structurelles spécifiques (i.e. celles dont la variation entraîne discontinuité et irréversibilité dans le fonctionnement du système), et (4) synthèse des caractéristiques du régime. On peut dire qu'on en est resté en gros au second stade dans les analyses conventionnelles.

${ }_{9} \quad$ La notion de pattern ne dépasse guère le niveau superficiel de la similarité et de la dissimilarité: il s'agit purement d'un arrangement au niveau de la classification et du rangement. Le simple fait d'observer un pattern ne révèle que peu de choses quant à la nature des forces sous-jacentes. Au contraire, la notion de réseau («network»), tout au moins dans la définition contrastée qu'en donne S.F. Nadel - «the interlocking of relationships whereby the interactions implicit in one determine those occuring in others" (The Theory of Social Structure (London, 1957), 14-17) - nous rappelle que les sous-procès sont des noeuds de relations et que l'intégration des sous-procès, c'est l'intégration de relations en des réseaux qui définissent la nature de la trame informationnelle et institutionnelle de la socio-économie. Les patterns observés ne sont que le résultat, l'effet d'écho observable de cette intégration de réseaux de relations. Voir G. Paquet, «A Meso-Economic Perspective of Structural Transformation", in I. Dobosi, ed., The Economic Choice of Small Countries in a Changing World Economic Environment (Budapest, 1981), 27-37.

10 Cette rationalité de fonctionnement d'un sous-procès ou du procès diffère complètement de la rationalité de comportement qui est utilisée dans les débats conventionnels en sciences humaines. On peut dire de la première qu'elle est une rationalité substantifique («Substantive Rationality»), par rapport au caractère formel de la seconde. Il s'agit d'une vieille distinction de Max Weber reprise avec profit par A.G. Ramos, The New Science of Organizations (Toronto, 1981), dans un effort pour produire une théorie substantifique des organisations. Cette distinction rejoint la différence que suggérait Karl Polanyi entre les sens formel et substantifique de l'économie. Cette rationalité de fonctionnement qui est au centre des sous-procès fait que ces derniers échappent à un simple rôle instrumental et se développent en un noeud de relations dont la dérive ne correspond pas à une manipulation non plus qu'au simple hasard. Voilà d'ailleurs qui correspond à la notion de changement social chez Popper (B. Magee, Popper, London, 1975). 
comme un «système d'action concret» au sens de Crozier et de Friedberg ${ }^{11}$.

La trame institutionnelle d'un sous-procès ou d'un sous-jeu dans une période ou dans un lieu donné ne constitue pas simplement un ensemble de règles du jeu préexistantes et pesant comme autant de contraintes sur le comportement des agents. Les institutions émanent d'une situation équivalente à un jeu à $n$ personnes avec possibilités de coopération et d'alliances. Cette émanation organique qu'est l'institution fait partie de la solution de ce jeu, même s'il ne s'agit pas d'un construit dont l'architecture résulterait par négociation des marchandages délibérés entre agents économiques. Comme le dit si bien Karl Popper, "only a minority of social institutions are consciously designed while the vast majority have just 'grown' as the undesigned results of human actions» ${ }^{12}$.

Chacun des sous-procès est constitué d'un regroupement de ces jeux arrimés les uns aux autres et la totalité des sous-procès forme un ensemble complexe de ces systèmes de jeux articulés. Un régime de fonctionnement sera donc un ensemble cohérent et robuste de ces solutions institutionnelles constituant un type de «grand jeu». Ce qu'il faut tenter d'identifier, c'est le leitmotiv qui démarque les institutions de l'époque, le code de la trame institutionnelle qui caractérise le grand jeu. Quand on passe d'un régime à un autre, du «jeu féodal» au «jeu capitaliste» (pour reprendre le raccourci de Crozier et Friedberg), ce n'est pas simplement «par un changement des règles du jeu féodal qui aurait donné plus de liberté au vassal face à son seigneur ou qui aurait renversé les rôles. Il s'est effectué par l'apprentissage d'un nouveau type de jeu complètement différent. Le jeu capitaliste n'est pas une amélioration du jeu féodal, c'est un jeu d'une autre nature." ${ }^{13}$ Une discontinuité marque le passage d'un jeu à un autre.

Les sources et les causes de ces passages d'un jeu à un autre, il ne faut pas les chercher uniquement à l'intérieur du réseau constitué par le sous-procès ou dans l'interadaptation entre les sousprocès. Pour une socio-économie comme celle du Québec et du Canada, fragment colonial de l'univers atlantique, les chocs de l'extérieur auront un impact déterminant dans les glissements ou

11 «Nous pouvons donc définir finalement un système d'action concret comme un ensemble humain structuré qui coordonne les actions de ses participants par des mécanismes de jeux relativement stables et qui maintient sa structure c'est-à-dire la stabilité de ses jeux et les rapports entre ceux-ci, par des mécanismes de régulation qui constituent d'autres jeux». (M. Crozier et E. Friedberg, L'acteur et le système (Paris, 1977), 246).

12 K.R. Popper, The Poverty of Historicism (New York, 1964), 65.

13 M. Crozier et E. Friedberg, op. cit., 332. 
les sauts d'un régime de fonctionnement à un autre. Il faudra donc non seulement faire la part des conséquences non prévues et non voulues des actions humaines à l'intérieur de la socio-économie, mais aussi la part des forces exogènes transformant les jeux de l'extérieur, en particulier les pulsions venant de l'Empire français, de l'Empire britannique et des États-Unis.

Chacun des sous-procès commanderait une analyse en profondeur afin d'en déceler le code et de profiler les configurations de causes qui, au niveau des réseaux de relations qui le soustendent, en ont entraîné la dérive, le changement. C'est un exercice auquel nous nous sommes livrés en détail pour ce que nous allons nommer la «seconde grande discontinuité» dans la prochaine section ${ }^{14}$. Il n'est évidemment pas question d'étudier ici en détail les six sous-procès pour chacun des régimes de fonctionnement non plus que de scruter la nature précise des causes de chaque discontinuité. Notre propos est moins ambitieux: tout au plus s'agit-il «to map out the problem area and thus prepare the ground for its empirical investigation by appropriate methods» ${ }^{15}$.

Pour éviter une prospection trop elliptique, nous avons, autant que faire se peut, tenté dans l'examen des différents régimes de fonctionnement de faire référence à trois éléments essentiels: une structure, une technologie, une théorie. La structure définit les rôles, les valences des acteurs ainsi que les relations entre eux; la technologie, l'ensemble des procédures et pratiques à travers lesquelles le jeu s'accomplit; la théorie, l'ensemble des règles épistémologiques qui sert aux acteurs pour interpréter les réalités internes et externes et les aide à y faire face. Ces trois dimensions essentielles sont interdépendantes: un changement dans une dimension déclenche un glissement dans les autres et, par voie de conséquence, peut se répercuter sur tout le système ${ }^{16}$.

14 Voir en particulier G. Paquet et J.-P. Wallot «Groupes sociaux et pouvoir: le cas canadien au tournant du XIXe siècle» [«Groupes sociaux»], RHAF, 27,4 (mars 1974): 509564 , où nous analysons l'écologie des groupes sociaux et leurs motivations.

15 S.F Nadel, op. cit., 1.

16 C'est une approche développée par D.A. Schon, Beyond the Stable State (New York, 1971), chap. 2. Pour Schon, il est futile de rechercher une "cause» pour laquelle un système ou un procès revêt un tour ou des caractéristiques données. C'est dans l'interaction 
Une telle approche devrait nous aider à éviter un travers important dans l'analyse économique des institutions, celui de les ramener toutes à une logique du marché («Public Choice»): l'architecture des institutions peut refléter des logiques autres que celle du marché ${ }^{17}$. De plus, le fait de tenir compte explicitement des perceptions du monde et des valeurs inévitablement liées à «la théorie» du monde dans lequel se meuvent les acteurs, nous amènera à déborder les explications strictement fonctionnalistes ${ }^{18}$.

des structures formelles et informelles, des perceptions et des valeurs qui habitent ceux qui vivent dans le système, et des outillages matériels et mentaux dont ils usent que se crée le caractère original du procès et que se développe la capacité de ce procès à durer, à se défendre contre les menaces de l'extérieur. Par un certain conservatisme dynamique, sorte d'inertie créatrice, des institutions peuvent survivre longtemps après que les situations qui les ont engendrées ont disparu. On a voulu expliquer ce conservatisme dynamique simplement par l'intérêt particulier des individus qui voient le lien entre leur bien-être et la survicance du système. Mais de dire Schon, cette interprétation n'est pas entièrement satisfaisante et n'éclaire pas le caractère souvent non rationnel de la résistance au changement. Si on admet que le système n'est pas pour ses membres «only sources of livelihood, protection against outside threat and the promise of economic security, but a framework of theory, values and related technology which enables individuals to make sense of their lives», cette résistance devient alors plus facile à comprendre, parce que toute menace à ce système pèse aussi sur ce cadre de référence (p. 51). Voilà pourquoi il nous a paru important de retenir ces trois dimensions dans notre survol de l'expérience de la socio-économie québécoise, puisque c'est dans cette interaction que s'explique la robustesse d'un régime de fonctionnement et que peuvent se lire les commencements de son changement.

17 Karl Polanyi insistait sur trois grands principes de structuration sociale (réciprocité, redistribution et échange marchand). Or il y a eu dans le mouvement néo-institutionnel une tendance très forte à tout interpréter par l'échange marchand. Le politique et le social sont réduits à cette logique (v.g. G. Tullok, «Economic Imperialism», in J.M. Buchanan et R.D. Tollison, ed., Theory of Public Choice, Ann Arbor, 1972). Sans vouloir minimiser l'utilité de ces travaux, il nous a paru plus utile de garder ouverte la possibilité que des logiques autres que celle du marché puissent prédominer dans la création et le maintien d'institutions, que ce soient les autres possibles suggérés par Polanyi ou même des dimensions isonomiques (orientation communautaire) ou phénonomiques (orientations de développement personnel) (comme le suggère A.G. Ramos, op. cit., chap. 7).

${ }_{18}$ Le but est évidemment de discerner la logique d'un système d'action concret, le code d'un régime de fonctionnement. Or on ne peut percevoir ce dernier comme une métainstitution délibérément construite ni en dériver les contours strictement à partir d'une rationalité formelle/fonctionnelle. Tout au moins, les tentatives en ce sens comme celles de Davis et North n'ont pas produit des résultats très impressionnants: ils l'ont admis d'ailleurs eux-mêmes dans la conclusion de leur travail de 1970. Il y a même danger que tout ce que puisse suggérer une analyse de ce genre ne soit qu'une sorte de rationalisation des innovations institutionnelles (au niveau des petits arrangements) sans arriver à donner une explication des contours de l'environnement institutionnel plus large (i.e. l'ensemble des règles du jeu, les droits de propriété, la monnaie, l'État, etc.). Il semble que c'est seulement en réintégrant à l'analyse l'univers des valeurs qu'on pourra rejoindre cette rationalité «substantifique» («substantive rationality») qui tient la clé d'une bonne compréhension des régimes de fonctionnement de la socio-économie québécoise. Voir A.G. Ramos, op. cit., chap. 2 . 


\section{Quatre grandes discontinuités / cinq régimes de fonctionnement: Un scénario}

Du natura non facit saltum à la loi de la continuité de Leibnitz, au lex continui qu'Alfred Marshall posait en épigraphe à ses Principles of Economics et jusqu'au crédo de la plupart des historiens contemporains, s'impose le dominium de la continuité. Pourtant, il s'agit là bien plutôt d'un outil forgé par l'artisan que d'une réalité inscrite dans le matériau historique ${ }^{19}$. En fait, la nature accomplit des sauts qu'on peut observer très clairement: par exemple, lorsque la température de l'eau tombe en dessous de $32^{\circ} \mathrm{F}$ ou dépasse les $212^{\circ} \mathrm{F}$. À ce moment précis, le changement quantitatif continu donne lieu à une transformation qualitative discontinue. C'est l'analogie qu'utilise Edward Tiryakian pour établir la différence entre changement social continu et changement sociétal discontinu ${ }^{20}$.

Notre lecture de l'expérience de la socio-économie québécoise ne conduit pas à nier le changement social, mais bien plutôt à souligner le changement sociétal à partir d'une grille d'analyse intégrant les six sous-procès évoqués dans la section 2 et trois de leurs dimensions essentielles (technologie, structure, théorie). Le bond ou la mutation n'est pas nécessairement déclenché toujours par le même sous-procès non plus que par la même dysfonctionnalité entre structure-technologie-théorie. La grille retenue présente l'avantage de pouvoir cerner des dynamiques de rupture issues de sources différentes.

Pour fixer les idées, posons dès le départ que notre analyse dégage quatre grandes discontinuités. Cette hypothèse émane de notre lecture du dossier documentaire et d'un premier effort de prospection à l'aide de notre grille d'analyse. Ces quatre discontinuités se situent au tournant du XVIIIIe, au tournant du XIXe, dans le troisième tiers du XIXe et dans le troisième tiers du XXe siècle. Elles démarquent cinq régimes de fonctionnement que nous avons baptisés en raccourci: économie-comptoir, socio-économie duale, capitalisme commercial, capitalisme industriel, socioéconomie d'information. Bien entendu, les ruptures ne sont jamais totales et elles s'étalent sur des périodes plus ou moins longues, la «viscosité» (Labrousse) des sous-procès suscitant des délais dans la propagation des effets d'une dimension à une autre.

38.

19 A. Gerschenkron, Continuity in History and Other Essays (Cambridge, 1968),

20 E.A. Tiryakian, «A Model of Societal Change and Its Lead Indicators», in S.Z. Klausner, ed., The Study of Total Societies (New York, 1967), 73. 
Ces dates et ces étiquettes n'explicitent pas suffisamment le changement sociétal qu'elles démarquent et qualifient; mais même dans cette formulation sommaire, elles ont servi à la mise au point d'un test de plausibilité. On pourra rétorquer que cette forme assez floue immunise notre hypothèse contre toute réfutation. En fait, tel n'est pas le cas. Comme nous l'avons exposé ailleurs, notre problématique n'est immunisée que contre la réfutation brutale et la «cruauté méthodologique» ${ }^{21}$. Nous l'avons confrontée à un compendium de réalités, à un état de la question en histoire économique et sociale; et nous nous sommes contentés - comme nous le suggérions dans notre article de 1979 - de vérifier si notre problématique déclarait impossibles des états de faits observés par les experts dans le domaine. Pour ce premier test, nous avons fait appel à 59 spécialistes que l'un de nous a interviewés de façon intensive entre les mois d'août 1980 et d'avril $1981^{22}$. Le résultat de ces entrevues a été diffusé sur les ondes de Radio-Canada ${ }^{23}$.

Sans prétendre nous restreindre à ces seuls résultats, il reste que ce test peu conventionnel de notre problématique en a révélé clairement la capacité à guider effectivement l'enquête ainsi que son pouvoir heuristique. On ne saurait détailler ici tous les éléments du dossier documentaire qui étayent le scénario proposé. Tout au plus les paragraphes qui suivent tentent-ils d'en montrer la plausibilité de façon assez générale. Il faudra un cadre plus ample

21 G. Paquet et J.-P. Wallot, «Pour une méso-histoire [...]», section 2.b.

22 Cette méthode assez peu conventionnelle a le mérite de provoquer directement les réactions des experts à l'hypothèse et de mettre à contribution les travaux les plus récents, même ceux qui n'ont pas encore été publiés. La liste des personnes interviewées (par ordre d'apparition au moment de la diffusion) est présentée ci-après. Plusieurs intervenants sont revenus plusieurs fois dans des émissions ultérieures à leur première apparition. La liste se lit comme suit: Jean-Pierre Wallot, Pierre Harvey, Alfred Dubuc, Frédéric Mauro, Denis Delage, Cameron Nish, Jacques Mathieu, Abraham Rotstein, Lucien Campeau, Jean-Luc Migué, Denis Monière, Jean Daigle, Louise Dechêne, Jean Hamelin, John Bosher, Pierre Tousignant, Fernand Ouellet, José Igartua, Léon Thériault, Del Muise, Foster Griezic, Almos Tassonyi, Antoine Lussier, Stanley Bréhaut Ryerson, Paul-André Linteau, John McCallum, Normand Séguin, Yolande Lavoie, Johanne Burgess, Bryan Young, Steven Langdon, Gilles Piédalue, Raymond Boily, Fernand Harvey, Hubert Charbonneau, Jacques Rouillard, Glen Williams, John Thompson, Yves Saint-Germain, Richard Clippingdale, François Vaillancourt, Bernard Bonin, Jorge Niosi, Andrée Lévesque, Blair Neatby, Carman Miller, Robert Armstrong, John Richard, Allan Moscovitch, Alvin Finkel, Caroline Pestieau, Gérard Bélanger, Irving Brecher, André Raynauld, Eric Kierans, Ralph Surette, Rolland Parenteau, Kimon Valaskakis, Fernand Martin.

23 Les émissions se sont échelonnées entre les mois de novembre 1980 et avril 1981. La transcription des entrevues était disponible auprès de la Société Radio-Canada en 1981: G. Paquet, Histoire économique du Canada (Société Radio-Canada, 1980-1981), 25 cahiers, 537 p. Les cahiers 1 à 3 portent sur l'économie comptoir, le cahier 4, sur la première discontinuité, les cahiers 5-6, sur l'économie duale, les cahiers 7-8, sur la seconde discontinuité, les cahiers 9 à 11 , sur le capitalisme commercial, les cahiers 12 à 17 , sur la troisième discontinuité, les cahiers 18 à 24 , sur le capitalisme industriel, le cahier 25 , sur la quatrième discontinuité et la socio-économie d'information. 
et des études plus fouillées pour fonder une vérification moins elliptique ${ }^{24}$.

\section{a) Une socio-économie-comptoir}

La poignée de Français qui tentent d'implanter un poste permanent dans ce coin de l'Amérique du Nord-Est, au tournant du XVIIe siècle, font un trait d'union entre un système économique atlantique caractérisé et une socio-économie amérindienne elle aussi caractérisée. Ils établissent de fait un «comptoir» - «un établissement commercial d'une nation à l'étranger» (Larousse) selon l'expression de Marcel Trudel ${ }^{25}$. Et les formes d'organisation économique qui se succèdent tout au long du siècle peuvent être considérées comme autant d'armistices entre les forces émanant des deux milieux mis en contact par la soudure coloniale.

L'Atlantique, c'est le monde de la régulation économique mercantiliste. L'absolutisme, la vénalité des charges et le régime des guildes prédominent en France sous Henri IV au tournant du siècle. Plus tard, durant les règnes de Louis XIII et de Louis XIV, l'État français se dote d'un système de perception des taxes qui arrache à ses sujets de trois à quatre fois plus d'impôts per capita que l'État anglais ne réussit à le faire au même moment ${ }^{26}$. Dans ce contexte, l'octroi de monopoles à des compagnies privées apparaît comme une stratégie fiscale conçue pour gonfler à peu de frais les revenus de l'État. On a pu dire que sous l'administration de Colbert (1662-1683), pas moins de la moitié des revenus de l'État proviennent de l'octroi de chartes de monopoles et de cartels ${ }^{27}$. En d'autres mots, le Canada, «une création du capitalisme marchand, une région satellite subordonnée à la métropole» ${ }^{28}$, existe comme émanation du mercantilisme de la France, lui-même le produit des contradictions de la société métropolitaine en pleine transition du féodalisme au capitalisme ${ }^{29}$.

\footnotetext{
24 Dans les références à la transcription des émissions, nous identifierons les documents par deux chiffres: xx-zz, le premier renvoyant au cahier, le second, à la page.

${ }_{25}$ M. Trudel, Histoire de la Nouvelle-France, tome 2: Le comptoir 1604-1627 (Montréal, 1966).

${ }^{26}$ R.B. Ekelund jr et R.D. Tollison, Mercantilism as a Rent-Seeking Society (College Station, 1981), 78.

27 Ibid., 6.

28 L. Dechêne, Habitants et marchands de Montréal au XVIIIe siècle [Habitants] (Paris, 1974), 482.

29 Sur l'exportation des contradictions françaises et le mercantilisme, voir L. MacDonald, «France and New France: The Internal Contradictions» [«France»], CHR, 52 (1971): 121-143.
} 
Dans la partie nord-est de ce continent existe déjà, avant l'arrivée des Européens, une socio-économie qui implique peut-être un million d'habitants: le groupe algonkien au nord, des chasseurscueilleurs pour ce qui est du Canada actuel et des horticulteurs sur la côte atlantique; le groupe iroquoien autour des Grands lacs, des horticulteurs qui ne pratiquent la chasse et la pêche qu'accessoirement. Les Hurons (une branche de la famille iroquoienne) assurent le gros du commerce, de la Baie James aux Grands Lacs (D. Delage: 01.16-17).

Le comptoir articule ces deux «économies-monde» (Braudel). Trait d'union largement fondé sur le commerce des fourrures, il se révélera une aventure bien moins profitable qu'avaient pu le penser ceux qui avaient acheté le monopole. D'où la succession de formes d'organisation depuis les compagnies privées jusqu'au gouvernement royal, en passant par toute une gamme de formes intermédiaires du type coopératif et «société de la Couronne». C'est que la greffe de ce comptoir «prend» mal: contraintes climatiques importantes; mauvaise insertion de la Nouvelle-France dans les réseaux du commerce atlantique à cause de l'exiguïté de ses ressources naturelles en regard de la demande sur le marché impérial; insignifiance du peuplement; concurrence efficace des colonies britanniques de la Nouvelle-Angleterre (et, pour un temps, de la «Nouvelle-Hollande»); guerres économiques entre Amérindiens qui précipitent l'extinction des Hurons, alliés des Français, et obligent ces derniers à déployer leur empire vers l'intérieur du continent pour aller quérir la fourrure, lors même que leurs concurrents attirent des intermédiaires à moindres frais jusqu'à Albany.

Même après 1660, l'application des plans détaillés de Colbert et de Talon se soldent par des résultats décevants. La lancée d'explorations et d'essaimage de postes à l'échelle continentale - «bel exploit... disproportionné aux ressources de la population canadienne» - ${ }^{30}$ s'enlise dans la crise des fourrures, à la fin du siècle, alors que l'échec ou la stagnation des initiatives de diversification économique sont tributaires, pour une large part, de leur caractère un peu artificiel et prématuré. L'appareil de réglementation trop lourd destiné à ériger une "société préfabriquée» (Sigmund Diamond) traduit l'esprit du mercantilisme triomphant en France. Commerce, tenure de la terre, répartition spatiale des hommes, comportement démographique, religion, droit civil, autant de dimensions parmi d'autres qu'on veut régir dans le but de mobiliser toutes les énergies à l'intérieur de cette «organisation sociale». 
Mais ce «plan» ${ }^{31}$, on veut l'imposer à une population qui a franchi l'Atlantique à grands risques parce qu'on lui a promis ou qu'elle espérait la promotion sociale. Les restrictions à la chasse et à la pêche, les amendes frappant les célibataires ou leurs parents, les tentatives de regrouper la population dispersée en villages, les velléités de multiplier les corvées provoquent des réactions vives contre l'encadrement: par exemple, les habitants refusent de se regrouper en villages, de payer la dîme s'ils le peuvent. Ils fuient la discipline sociale, colonisent où bon leur semble ou se tournent vers l'Ouest et le monde des fourrures de façon à échapper pour une bonne part au contrôle des autorités.

À la fin du XVIIe siècle, il ne restera plus grand-chose des plans de Talon, sauf l'apport de sang neuf: seule sa politique démographique aura donné quelques résultats, notamment grâce à l'installation des 800 hommes du régiment Carignan-Callières et à la venue des «filles du roi». Et il aura fallu vingt-cinq autres années avant que cette injection soudaine (et modeste) de colons n'assure la présence de plus de 10000 personnes, dont une forte proportion de jeunes adultes déjà en ménage ou cherchant à s'établir ( $\mathrm{J}$. Mathieu: 04.2-3).

Au cours du dernier quart du XVIIe siècle, s'accroissent non seulement la taille de la population, mais aussi l'acuité des problèmes dans le commerce des fourrures. Des formes embryonnaires, mais nouvelles, d'organisation économique commencent à émerger. On passe d'une économie de troc, où le numéraire est la peau de castor, à une économie où devises et lettres de change s'insinuent elles aussi. Le manque habituel de devises que draîne vers la mère patrie un déficit chronique de la balance commerciale, aboutit au développement d'un système complexe de crédit (L. Dechêne: 03.17-19) et même, en 1685, à la première expérience d'une monnaie fiduciaire, la monnaie de cartes: des cartes à jouer sont coupées en morceaux, portent la signature de l'intendant de Meulles et circulent comme de la monnaie.

Le comptoir se peuple peu à peu et sa base économique, la fourrure, s'effrite à cause de la surproduction et de l'engorgement du marché. La société préfabriquée qu'on avait envisagée comme organisation où chacun aurait sa place, apparaît de plus en plus comme une société éclatée qui échappe au contrôle réglementaire et dévie des plans originels. Peu d'échanges marchands peuvent s'établir à l'intérieur de la colonie, tant entre habitants, petits pro-

31 Pour une évocation récente de ce «plan», voir S. Courville, L'habitant canadien et le système seigneurial, 1627-1854, thèse de Ph.D. manuscrite, Département de géographie (Un. de Montréal, 1979). 
ducteurs «parcellaires» ou «indépendants», qu'entre eux et des artisans isolés ${ }^{32}$. Les tensions, entre les contraintes géotechniques de l'Amérique du Nord-Est et les théories européennes qui ont voulu mouler l'organisation économique de la Nouvelle-France, sont telles que des ajustements structurels s'imposent pour les accommoder.

\section{b) Une première grande discontinuité}

Avec le tournant du XVIIIe siècle, la conjonction d'une croissance rapide de la population et de la crise des fourrures déclenche la première discontinuité. D'un comptoir tourné avant tout vers la traite des fourrures sort une colonie à deux versants: un versant atlantique, toujours rattaché aux grands commerces, où s'accomplit une certaine accumulation du capital marchand, et un versant continental, enraciné dans l'agriculture et dans un embryon de marché local plus diversifié. Cette transformation du Canada «n'est pas une diversification qui vient de décisions gouvernementales, par en haut; c'est la vie dans le pays, les ressources du pays, l'organisation de la structure économique qui oblige chacun des individus à se trouver une activité qui lui permette de survivre» (J. Mathieu: 04.3). Cette amorce de diversification, davantage «naturelle» et largement tributaire des capitaux privés, répond aux besoins concrets d'un marché local naissant et d'un marché international qui s'ouvre (Antilles, Ile Royale et Louisbourg). À côté du secteur dominant (fourrures) surgissent ou croissent de nouveaux secteurs commerciaux et de petites industries locales: v.g. pêcheries sédentaires, érection de moulins à farine et de scieries, exportations de céréales, de bois, de chanvre, d'huile de phoque, de ginseng. L'État commence à encourager l'amélioration des voies de communication, sans compter ses investissements massifs, même s'ils sont parfois peu productifs ${ }^{33}$.

Il en résulte deux réseaux de fonctionnement en parallèle que Louise Dechêne a bien caractérisés ${ }^{34}$. D'une part, les habitants vivent en régime de quasi-autosuffisance dans les campagnes, mal-

32 L. Dechêne, Habitants, 186-187, 230, passim; D. Monière, «L'utilité du concept de mode de production des petits producteurs pour l'historiographie de la NouvelleFrance» [«L'utilité»], RHAF, 29,4 (mars 1976): 500.

33 G. Frégault, Le XVIIIe siècle, $374 \mathrm{ss}$, 381šs; J. Hamelin et al., Histoire du Québec (Toulouse-Saint-Hyacinthe, 1976), chap. VI; J. Mathieu, "L'Échec de la construction navale royale à la fin du régime français», Communications. Société historique du Canada (1968): 33; idem, La construction navale à Québec, 1739-1759 (Québec, 1971), surtout la conclusion; W.J. Eccles, «The Social, Economic, and Political Significance of the Military Establishment in New France», CHR, 52 (mars 1971): 1-32.

34 L. Dechêne, Habitants et marchands, conclusion. 
gré certaines retombées des exportations ponctuelles de céréales; d'autre part, dans les villes, s'intensifient les rapports de la population au marché atlantique et aux grands commerces. En exagérant, on peut parler de deux structures sociales, de deux organisations économiques, de deux formes de production et d'échange, de régimes financiers séparés même. Cette dichotomie de la socioéconomie québécoise - côté castor, côté blé - fait émerger, à côté du monde du comptoir, une paysannerie qui prend racine au tournant du XVIIIe siècle (J. Hamelin: 04.7). Denis Monière a attaché à ce second monde l'étiquette du «mode de production du petit producteur» ${ }^{35}$.

On a trop caricaturé les voies parallèles de ces deux versants de la colonie après les avoir longtemps ignorées. Certes, il n'y a pas en Nouvelle-France une agriculture autarcique; mais les régimes de fonctionnement dans les deux secteurs sont suffisamment contrastés pour qu'on puisse parler d'acteurs distincts, de groupes sociaux différents, de productions et d'échanges empruntant des modes différents d'actualisation. Cette mutation s'accomplit assez rapidement au tournant du XVIIIe siècle, moment où semblent se bousculer un ensemble de changements: d'où l'opinion de Frégault selon qui on peut parler alors de maturité naissante ${ }^{36}$.

\section{c) Une socio-économie duale}

Tout au long du XVIIIe siècle, l'expansion du secteur rural, l'augmentation des effectifs de la paysannerie et du monde des petits producteurs en général font surgir des acteurs nouveaux sur la scène économique, des relais avec le monde du grand commerce (v.g. les marchands de campagne). D'autre part, le commerce des fourrures pénètre lui aussi de plus en plus profondément dans le terroir à mesure qu'il lui faut aller chercher la fourrure de plus en plus loin au coeur du continent et utiliser une main-d'oeuvre et des provisions plus abondantes. Cependant, cette réalité qu'on a parfois considérée comme un procès d'interpénétration progressive ne s'accomplira vraiment qu'à la toute fin du siècle.

Entre-temps, c'est un double approfondissement de deux socio-économies en coexistence, dont les articulations mettent du temps à se définir et à se consolider. Il y a plutôt des débordements de chaque secteur sur le territoire de l'autre: la population double entre 1700 et 1730; la superficie des terres en culture quadruple

35 D. Monière, «L'utilité».

36 G. Frégault, Le XVIIIe siècle, 281. 
entre 1706 et 1739 (P. Tousignant: 05.2); on commence à exporter des surplus agricoles vers Louisbourg et les Antilles dont le commerce avec la Nouvelle-France se complexifie ${ }^{37}$; les moulins se multiplient et les meuniers s'enrichissent dans la mesure où les surplus exportables (et les marchés) deviennent suffisants pour supporter ces petites entreprises «commerciales». Mais la rareté de la maind'oeuvre et l'abondance des terres maintiennent la nécessité d'accorder aux artisans et aux habitants le libre accès à leurs moyens de subsistance (liberté de travail, terres), ce qui freine la différenciation sociale et assure la persistance d'une large couche de petits producteurs indépendants ${ }^{38}$.

Par ailleurs, du côté atlantique, les fourrures ne constituent plus le seul espoir des architectes de la vie économique de la Nouvelle-France. Ainsi, l'intendant Hocquart cherche à exploiter les ressources forestières du pays et son caractère atlantique en misant sur la construction navale; l'entrepreneur Cugnet s'oriente vers l'exploitation du minerai de fer à Trois-Rivières ${ }^{39}$. On conçoit même une vocation atlantique et politique pour la NouvelleFrance débordant la pêche et la cueillette des fourrures et débouchant jusque sur l'industrie lourde (sans parler de la guerre).

Ces deux versants de la socio-économie du Québec au XVIIIe siècle ne réussissent pas tellement à s'interpénétrer: ils ont différents réseaux de crédit, de monnaies même, de structures sociales. On peut même affirmer que le rythme de la vie économique est différent d'un pan de l'économie à l'autre. L'administration financière de la Nouvelle--France conduit aux abus les plus flagrants et engendre des taux d'inflation galopants quand les autorités se remettent à faire une politique monétaire expansionniste avec de la monnaie de cartes. Mais alors que l'inflation déchaînée mise en lumière par Jean Hamelin ${ }^{40}$ enraye le cours normal de l'activité économique dans le secteur atlantique, on observe un niveau de vie assez confortable et stable dans l'arrière-pays ${ }^{41}$. Même la guerre de la Conquête, avec ses poussées de prospérité et de misère réparties inégalement entre les diverses couches sociales, ne modifie pas vraiment de façon durable la matrice économique canadienne.

Les grands changements politiques découlant de la Conquête ne brisent pas non plus cette dualité, bien au contraire. Trevor

37 J. Mathieu, Le commerce entre la Nouvelle-France et les Antilles au XVIIIe siècle (Montréal, 1981).

38 L. MacDonald, «France», 125ss, 134ss; L. Dechêne, Habitants, 485ss.

39 Voir J. Mathieu, La construction navale à Québec; C. Nish, François-Étienne Cugnet. Entrepreneur et entreprise en Nouvelle-France (Montréal, 1975).

$4_{0}$ J. Hamelin, Économie et société en Nouvelle-France (Québec, 1960), chap. I.

${ }_{41}$ F. Ouellet, «La mentalité et l'outillage mental de l'habitant canadien-français: à propos d'un document sur l'encan"), Bulletin des recherches historiques (1956): 131-139. 
Denton a montré comment ils ne bouleversent pas la vie des habitants des campagnes et se concrétisent simplement dans le remplacement de l'appareil d'institutions dans le versant atlantique de la socio-économie $^{42}$. Il y a substitution d'un mercantilisme à un autre et d'une population nouvelle de marchands et hauts fonctionnaires britanniques (avec leurs réseaux financiers et informationnels propres, branchés sur les réalités métropolitaines, et un accès privilégié au pouvoir) à une autre. Certes, la naissance (même lente) du Canada anglais n'est pas un fait inimportant. Mais les lendemains de Conquête n'apportent pas de rupture fondamentale immédiate dans la socio-économie (F. Ouellet: 06.8) au niveau des structures formelles externes et internes ${ }^{43}$.

L'économie duale d'avant 1760, complétée par des secteurs satellites encore modestes, reste en place après la Conquête. Les structures économiques demeurent essentiellement lẹs mêmes qu'auparavant. Le pôle dominant (la traite des fourrures) continue d'attirer les gros capitaux et demeure la source principale de l'accumulation du capital marchand ${ }^{44}$. Plus peut-être que dans les années 1730 , la traite s'articule un peu mieux à une commercialisation limitée et par à-coups des denrées agricoles (essentiellement le blé), par le truchement des pressions sur la main-d'oeuvre ${ }^{45}$. Toutefois, au niveau du personnel en haut de la pyramide sociale, la bourgeoisie britannique s'impose assez rapidement et en vient à dominer l'ensemble du commerce international de la colonie. La dualité économique se double donc d'une dualité socio-ethnique et politique qui tend déjà à se figer, malgré quelques alliances tactiques contre un gouvernement parlementaire colonial ${ }^{46}$. Cette dua-

42 T. Denton, «The Structure of French-Canadian Acculturation, 1759 to 1800 », Anthropologica, 8 (1966): 29-42.

43 G. Bernier soutient que la Conquête entraîne une rupture fondamentale par suite de l'intrusion d'un mode de production capitaliste plus avancé au sein d'un mode de production à dominante féodale. Toutefois, outre les postulats non démontrés sur le régime français et les comparaisons implicites entre périodes différentes, l'auteur n'apporte aucun élément de preuve qu'il y ait vraiment des modifications importantes dans les structures économiques et le comportement des marchands (ou des habitants) avant la seconde discontinuité et plus particulièrement l'explosion du commerce du bois. («Sur quelques effets de la rupture structurelle engendrée par la Conquête au Québec: 1760-1854», RHAF, 35, 1 (juin 1981): 69-95).

44 L. Dechêne, Habitants, 483; F. Ouellet, "Dualité économique et changement technologique au Québec (1760-1790)», Histoire sociale, 9 (1976): 256, 296.

${ }_{45}$ F. Ouellet, "Dualité», 258-259, 269; idem, Histoire économique et sociale du Québec, 1760-1850 (Montréal, 1966), chap. III, IV et V.

${ }_{46}$ Sur ces alliances et leurs ambiguités, voir $\mathrm{P}$. Tousignant, La genèse et l'avènement de la Constitution de 1791 , thèse de $\mathrm{Ph}$.D. manuscrite, Histoire (Université de Montréal, 1971), 255-349; J.-P. Wallot, Un Québec qui bougeait. Trame socio-politique au 
lité économique, socio-ethnique et politique encore mouvante préfigure déjà le duopole social des décennies subséquentes ${ }^{47}$.

\section{d) Une seconde grande discontinuité}

L'essentiel de la seconde discontinuité, c'est la pénétration beaucoup plus rapide et profonde du marché conquérant: ce dernier accélère la décomposition des structures duales précédentes comme de leurs supports et l'intégration de la socio-économie en un tout régi par ses propres lois (G. Paquet et J.-P. Wallot: 07 et 08. Passim). Ce que les anciennes institutions, maintenant remises en cause, et les vestiges du féodalisme n'avaient pu mettre en oeuvre, selon les mots d'Adam Smith, "the silent and insensible operation of foreign commerce and manufactures gradually brought about» ${ }^{48}$. Cette rupture, que nous avons qualifiée ailleurs de «restructuration et de modernisation» de l'économie et de la société québécoises ${ }^{49}$, se consomme au tournant du XIXe siècle. La structure sociale se complexifie avec la montée de groupes nouveaux (petits entrepreneurs, professions libérales, classes laborieuses davantage caractérisées) et la stagnation d'autres (seigneurs), au moment même où les acteurs sociaux tentent de redéfinir et d'ex-

début du XIXe siècle (Montréal, 1973), 255-349. L'étude des causes de l'élimination de la bourgeoisie canadienne-française par la bourgeoisie anglo-saxonne dans le grand commerce entre 1760 et 1780-1790, dépasse largement les cadres du présent article. Personne ne conteste le fait brutal, mais les uns l'attribuent aux changements dans les institutions et les hommes en provenance d'une nouvelle métropole, avec des conséquences ethniques et sociales (M. Séguin, M. Brunet, G. Bourque), d'autres, aux déficiences économiques, techniques et psychologiques de la bourgeoisie canadienne-française (F. Ouellet, J. Hamelin, G. Bernier, D. Creighton). D'autres encore font ressortir les difficultés croissantes des marchands canadiens dans un réseau commercial et informationnel étranger (J. Igartua, D. Miquelon, G. Paquet et J...P. Wallot). Quelles qu'en soient les causes, les historiens s'accordent pour constater l'instauration graduelle, plus ou moins rapide, d'une dualité sociale et ethnique qui n'atteindra son paroxysme que dans les premières décennies du XIXe siècle.

47 G. Paquet et J.-P. Wallot, «Groupes sociaux», section 5.

48 H. A. Innis, "The Penetrative Powers of the Price System», in Essays in Canadian Economic History (Toronto, 1956), 254.

49 G. Paquet et J.-P. Wallot, "Le Bas-Canada au début du XIXe siècle: une hypothèse» [«Le Bas-Canada»], RHAF, 25, 1 (juin 1971): 39-61. On trouvera des statistiques et des développements pour étayer cette hypothèse dans idem, "Aperçu sur le commerce international et les prix domestiques dans le Bas-Canada (1793-1812)», RHAF, 21, 3 (décembre 1967): 447-473; idem, "Crise agricole et tensions socio-ethniques dans le Bas-Canada, 18021812: éléments pour une ré-interprétation», RHAF, 26, 2 (septembre 1972): 185-237; idem, «The International Circumstances of Lower Canada, 1786-1810: Prolegomenon», [«International Circumstances»] CHR, 53 (1972): 371-401; idem, Patronage et pouvoir dans le Bas-Canada (1794-1812). Un essai d'économie historique [Patronage] (Montréal, 1973). Voir aussi F. Ouellet, Histoire économique et sociale du Québec, 1760-1850, ch. V, VI, VII et VIII. 
pliciter les règles du jeu politique et social dans un contexte mondial agité (guerres de la Révolution et de l'Empire). C'est aussi l'apparition de la corporation dans ses formes embryonnaires comme d'un système financier et bancaire, l'élargissement de la matrice de développement économique et l'omniprésence des signaux du marché dont les pulsions animent même les campagnes. En un mot, la production devient mercantile. Le marché a commencé à s'approprier enfin la terre comme il le fera, plus tard, pour le travail, ce qui a pour effet la transsubstantiation de la socio-économie: «... to include them in the market mechanism means to subordinate the substance of society itself to the laws of the market». "A chain reaction was started - what before was merely isolated markets was transmuted into a self-regulating system of markets. And with the new economy, a new society sprang into being. $\gg{ }^{50}$ Aussi l'histoire du Canada jusqu'à la Confédération est-elle, à bien des points de vue, le déroulement du conflit entre le marché et les obstacles qui le freinent, le procès d'agrégation des fragments de l'Amérique du Nord britannique en un marché «national».

À la fin du XVIIIe siècle coïncident une série de ruptures internes et externes: le dépeçage de l'ancien «empire naturel» du SaintLaurent, l'arrivée des Loyalistes, les premiers projets de libreéchange avec les États-Unis (P. Tousignant: 07.6), la division de l'ancienne «Province of Quebec», d'une part ${ }^{51}$; les grands bouleversements qui secouent l'Occident, d'autre part, telles la «Révolution des Lumières», les Révolutions américaine et française, parties intégrantes selon R.R. Palmer de la «Révolution démocratique» d'Occident ${ }^{52}$, l'explosion démographique, l'ascension du capitalisme et de la bourgeoisie. Le Québec/Bas-Canada se métamorphose sous le dynamisme du marché atlantique et s'imbrique plus étroitement dans cet univers turbulent, dans ce système porteur d'informations, de marchandises, de capitaux, de techniques, d'hommes, d'idées, de valeurs et d'institutions. Longtemps alourdie par un large secteur de subsistance et diverses institutions (locales et impériales) peu propices au libre développement du capital, l'économie duale du XVIIIe siècle se disloque sous la force dissol-

50 K. Polanyi, The Great Transformation (Boston, 1957), 71; idem, «Our Obsolate Market Mentality», in Primitive, Archaic and Modern Economics (New York, 1968), 61.

51 Voir la thèse classique de D.G. Creighton, The Commercial Empire of the St. Lawrence (New Haven, 1937). Réédité en 1956 sous le titre The Empire of the St-Lawrence.

52 Sur la participation du Québec au bouillonnement idéologique du monde atlantique, voir les communications au colloque «Le Canada à l'époque de la «Révolution atlantique» "in Annales historiques de la Révolutión française, no 213 (juillet-sept. 1973): $321-435$. 
vante du marché et fait place à une configuration socioéconomique nouvelle en réponse au marché international ${ }^{53}$.

La population double entre 1790 et 1815 et un mouvement subit d'urbanisation se dessine à compter de 1805 à Québec et à Montréal, mouvement qui débouche sur l'amorce d'un marché de la main-d'oeuvre, sur la dissolution des anciens rapports "personnels» de travail et sur l'élaboration de réglementations diverses annonçant l'incorporation des municipalités.

Les exportations décuplent entre 1786 et 1810 , se multiplient par quatre entre 1807 et 1810 . La flambée des exportations de bois à compter de 1807, comme élément moteur du développement, s'accompagne d'une commercialisation sporadique des produits agricoles. Et malgré la chute de la traite des fourrures, les stimulis en provenance de l'Atlantique ont des effets d'entraînement en aval et en amont: la demande de main-d'oeuvre et de denrées agricoles augmente, fait hausser les prix et les salaires et tend à constituer un marché intérieur unifié (hormis pour l'arbitrage des distances). De nouveaux secteurs percent ou se développent, tels la construction navale, les scieries, la fabrication de mâts, de cerceaux et de tonneaux, de chandelles, de savon, de papier et de chapeaux, les forges et les meuneries. L'essaimage de petits entrepreneurs et la réorientation de l'entrepreneurship établi ${ }^{54}$ suscitent des associations nouvelles plus complexes, la rationalisation de la production (ententes pour certains contrats de l'armée, monopolisation dans l'imprimerie à Québec, constitution d'ateliers plus vastes), le passage de rapports paternalistes, entre maîtres, d'une part, compagnons et apprentis, d'autre part, à des rapports capitalistes d'employeurs-employés ${ }^{55}$, la spéculation foncière et la remise en question du régime seigneurial.

Une structure beaucoup plus complexe de groupes sociaux succède aux blocs antérieurs plus simples (A. Dubuc: 07.9): il faut souligner plus particulièrement la montée de la petite-bourgeoisie des membres des professions libérales et des marchands modestes (ruraux notamment) qui articulent le nouveau nationalisme canadien-français ainsi qu'un projet de société centré sur le développement du Québec même ${ }^{56}$.

53 G. Paquet et J.-P. Wallot, «Le Bas-Canada»: 43-49; idem, «International Circumstances»: 374-383.

${ }_{54}$ A.R.M. Lower, «The Trade in Square Timber», University of Toronto Economic Studies, 6 (1933): 40-61; D.G. Creighton, The Commercial Empire of the St.Lawrence, chap. IV et $\mathrm{V}$.

55 J.-P. Hardy et T. Ruddell, Les apprentis-artisans à Québec 1660-1815 (Montréal, 1977); P.-H. Audet, Apprenticeships in Montreal, 1792-1812, thèse de M.A. manuscrite (Concordia, 1973).

56 Voir G. Paquet et J.-P. Wallot, «Groupes sociaux et pouvoir»; idem, Patronage, 51-73 et chap. V. 
Après les deux solitudes (relatives) des années 1760-1792 éclatent ou s'exaspèrent des conflits autour du contrôle du pouvoir au sein d'un État polyèdre, luttes qui deviennent chroniques et aboutissent à une série de freinages systématiques: ceux-ci résultent du recoupement, de la superposition et de la conjugaison de divers niveaux de conflit (constitutionnel, social, ethnique, économique) et s'enveniment de l'exercice d'un fort patronage de rétribution et de distribution. L'État s'enlise dans un pat et ne peut jouer le rôle actif exigé par les changements économiques. Ses branches militaire et civile investissent beaucoup, mais les définitions des besoins sociaux prioritaires et les projets de réponse politique concurrents se neutralisent ${ }^{57}$.

C'est aussi l'élaboration d'un système financier embryonnaire (apparition des premières compagnies d'assurance, des «Army Bills» durant la guerre de 1812) et la création des premières banques (G. Paquet: 08.20) ${ }^{58}$.

Enfin, une différenciation sociale croissante se fait jour, tant à l'intérieur d'un même groupe social que par la poussée de groupes nouveaux. Les habitants et la plupart des salariés voient leur niveau de vie s'améliorer: les acteurs se spécialisent davantage et se procurent, grâce au surplus que leur assure leur production, un superflu lié à la fois au bien-être, au genre de vie tout autant qu'à la recherche d'un statut social ${ }^{59}$.

L'ensemble des interactions entre sous-procès et la simultanéité d'un grand nombre de changements en chacun décalquent la trajectoire d'une socio-économie qui change de rythme de croisière.

\section{e) Le capitalisme commercial}

Le procès de modernisation, au tournant du XIXe siècle, a laissé la socio-économie québécoise restructurée et transformée. Le marché a commencé l'unification des versants disjoints de l'ancienne économie duale. Les mécanismes autorégulateurs du marché triomphant achèveront la dislocation de l'ancienne société, mais son fonctionnement est entravé par une série de résistances: derniers soubresauts du mercantilisme, structures seigneuriales et éléments archaïques de la Coutume qui perdurent jusqu'au milieu du siècle, écologie dysfonctionnelle des groupes sociaux et paralysie de l'Etat bas-canadien jusqu'en 1840 .

Idem, Patronage, chap. II, IV et V.

58 G. Paquet et J.-P. Wallot, «Le système financier bas-canadien au tournant du 19ième siècle»" (à paraître).

59 Voir G. Paquet et J.-P. Wallot, «Les inventaires après décès à Montréal au tournant du XIXe siècle: préliminaires à une analyse», RHAF, 30,2 (septembre 1976): 163-221. 
Désormais, l'agriculture est rattachée au monde atlantique pour le meilleur ou pour le pire. Au début du XIXe siècle, ce lien profite aux habitants. Mais la dépression qui frappe la GrandeBretagne à compter de 1815-1820, la chute des prix agricoles, les barrières tarifaires en Angleterre («Corn Laws») et la concurrence accrue du Haut-Canada/Canada-Ouest, favorisée par le climat et la qualité d'un sol neuf, s'abattent sur une agriculture qui éprouve des difficultés croissantes dans les années 1820 et surtout 1830 (épidémies de la mouche à blé, manque de terres ou spéculation foncière, montée d'un prolétariat rural). Par ailleurs, la commercialisation de l'agriculture substitue aux débats relatifs à la rente foncière d'autres débats qui se concentrent plutôt sur les fluctuations des prix et, éventuellement, sur l'accès au marché de la terre. Si la ville de Québec croît et s'organise autour du bois et de la construction navale, comme l'a signalé Albert Faucher ${ }^{60}$, Montréal, pour sa part, confirme son rôle de plaque tournante au centre de ce capitalisme marchand: elle polarise l'espace commercial canadien de l'Ouest et du Sud. C'est le poste-relais privilégié dans cette longue chaîne de communication qui relie l'Angleterre et les régions les plus excentriques: chaîne de communication financière, bancaire et commerciale qui inféode la périphérie (y compris le HautCanada) à Montréal ${ }^{61}$. Les deux villes s'organisent et se dotent d'institutions municipales mieux adaptées à leur croissance et à leur rôle économique.

Le régime du capitalisme commercial n'implique pas la stabilité et il ne reste pas intact tout au long de cette période. Après le coup d'envoi au tournant du siècle, il envahit toutes les sphères d'activité de la société québécoise: la monnaie, la terre, les niveaux de vie sont assujettis de plus en plus aux aléas du marché ${ }^{62}$. Les banques se multiplient ${ }^{63}$, de nouvelles institutions financières surgissent, et les formes de sociétés par action se développent comme autant de réactions aux exigences nouvelles d'un système économique de risque.

L'intégration aux grands marchés mondiaux comporte aussi des risques importants: v.g. les effets de la chute des prix du blé sur

60) A. Faucher, «La construction navale à Québec au XIXe siècle: apogée et déclin», in idem, Histoire économique et unité canadienne (Montréal, 1970), 227-254. 1964).

6i G. N. Tucker, The Canadian Commercial Revolution, 1845-1851 (Toronto,

62 Comme le démontrent Innis et Polanyi. Voir notes 2, 17, 48 et 50.

63 R. Rudin, «The Montreal Banks and the Urban Development of Quebec, 18401914», communication à la Canadian Urban History Conference, Université de Guelph, 1977 (miméo). 
l'agriculture québécoise, les retombées des transferts de devises entre les États-Unis et la Chine sur la vie économique de la colonie (G. Paquet: 10.10-11), l'impact des tractations entre le ministre des finances de la Province du Canada et les banquiers de Lombard Street sur l'avenir canadien ${ }^{64}$. Mais il y a plus. Lentement, la concurrence tenace des États-Unis sape le rôle hégémonique de Montréal. Montréal perd la grande bataille pour le contrôle de l'arrière-pays contre New York: pas seulement la bataille géotechnique, où Montréal souffre de handicaps certains, mais la bataille économique qui, elle, dépend de toute la technologie sociale des institutions financières (où les avantages de New York sur Montréal sont évidents) ${ }^{65}$. Le procès d'intégration des marchés et des prix ne réussit donc pas à entraîner le Nord des États-Unis dans l'orbite du commerce bas-canadien, comme l'espèrent encore les marchands montréalais au milieu du siècle. Il tend toutefois à la structuration et à la consolidation d'un marché «canadien» grâce à l'Union de 1840 et à la Confédération de 1867.

Durant ce régime du capitalisme commercial, la démographie se déstabilise. Jadis, des politiques délibérées qui assignaient à chacun sa place dans la société préfabriquée dont on rêvait sous Louis XIV, cherchaient à attacher les habitants à la terre. Ceux-ci prirent racine dans le sol avec le temps et une paysannerie moyennement prospère occupe les campagnes au début du XIXe siècle. Mais, dans les décennies subséquentes, l'explosion démographique interne et la rareté croissante (réelle ou spéculative) des terres déclenchent un mouvement important d'émigration vers les ÉtatsUnis, au moment même où des vagues croissantes d'immigrants des Îles britanniques déferlent sur le Québec et le Haut-Canada. C'est le jeu de la chasse aux rentes attachées aux ressources immobiles par des populations mobilisables par leur déracinement même ${ }^{66}$.

Le jeu social lui-même se complique. À côté de grands commerçants du type plus traditionnel comme William Price, membre de la bourgeoisie commerciale régnante ${ }^{67}$, prospèrent des types

64 A. Faucher, Histoire économique et unité canadienne, 65-82.

65 G. N. Tucker, op. cit., chap. II.

66 G. Paquet et W. R. Smith, "L'émigration des Canadiens français vers les ÉtatsUnis, 1790-1940: problématique et coups de sonde» (à paraître). La terre ou le sous-sol offrent des possibilités de gains importants pour les facteurs de production mobile (travail et capital) qui acceptent de se déplacer vers leur espace. Cependant, cette chasse au surplus (ou rentes) se fait dans la concurrence, de sorte que ces rentes peuvent être dissipées par les activités des facteurs mobiles concurrents qui se les disputent. Voir J. M. Buchanan, R. D. Tollison et G. Tulloch, ed., Toward a Theory of the Rent-Seeking Society (College Station, 1980).

L. Dechêne, «Les entreprises de William Price, 1810-1850», Histoire sociale, 1 (1968): 16-52. 
nouveaux de bourgeois qui débordent le commerce pour s'adonner à la production industrielle: par exemple, John Molson qui représente bien cette nouvelle bourgeoisie encore atypique, ce groupe d'entrepreneurs industriels (A. Dubuc: 09.13ss). La bourgeoisie se fractionne en branches diverses. La trame sociale se complique sous l'impulsion des débuts de l'industrialisation et la construction des grands ouvrages d'infrastructure (canaux, routes, bientôt les chemins de fer), qui constituent une stratégie de défense contre l'envahissement de New York et provoquent l'émergence d'un prolétariat québécois encore limité ${ }^{68}$. Bel exemple d'une aventure capitaliste encore rationalisée dans un schéma mercantiliste: la technologie devance la théorie ${ }^{69}$.

Au Québec, des facteurs à la fois économiques et politiques déphasent le régime de fonctionnement par rapport à l'expansion économique remarquable du Nord-Est des États-Unis et du HautCanada. Le pôle de la croissance économique au Québec (le bois) n'aurait pas la puissance d'entraînement du blé sur l'ensemble de la société, d'où l'avance prise par l'Ontario (J. McCallum: 10.6ss et 11.9) ${ }^{70}$. D'ailleurs, dans toute l'Amérique du Nord, la prospérité et le développement agricoles se déplacent vers le «MiddleWest», et c'est l'industrialisation qui prend la relève en NouvelleAngleterre, mais pas encore au Québec. De sorte que ce dernier devient une zone relativement marginale dans l'espace économique du Nord-Est de l'Amérique du Nord ${ }^{71}$.

Une source de blocage plus importante peut-être, c'est le grippage d'un État enlisé dans des conflits internes qui l'empêchent d'intervenir à ce moment crucial d'ajustement au capitalisme commercial et qui opposent des stratégies économiques de développement clairement contradictoires: la bourgeoisie britannique mise sur un capitalisme commercial continental, la bourgeoisie canadienne-française, sur le développement local (Cahier 10). C'est donc avec un certain retard que la colonie vit cette évolution au XIXe siècle. La fin du mercantilisme (puis la fin de ce vain espoir

68 H. C. Pentland, «The Development of a Capitalistic Labour Market in Canada», Canadian Journal of Economics and Political Science, 25,4 (nov. 1959).

${ }_{69}$ A. Faucher, Québec en Amérique au XIXe siècle (Montréal, 1973), chap. III.

70) J. McCallum, Unequal Beginnings. Agriculture and Economic Development in Quebec and Ontario until 1870 (Toronto, 1980). Le nombre très élevé et la variété des études sur la socio-économie québécoise à partir des années 1840-1850 nous empêchent de citer systématiquement les auteurs sur chaque question. On se reportera, d'une part, à la liste des personnes interviewées en note 22 et, d'autre part, aux notes historiographiques de notre article de décembre 1979, en ce qui a trait au XIXe siècle, et aux cahiers 12 à 17, pour la troisième discontinuité. Voir aussi J. Hamelin et Y. Roby, Histoire économique du Québec, 1851-1896 (Montréal, 1971), et R. Durocher, P.-A. Linteau et J.-C. Robert, Histoire du Québec contemporain, tome I: De la Confédération à la crise (Montréal, 1979).

71 A. Faucher, Québec en Amérique au XIXe siècle. 
de draîner les commerces de l'arrière-pays via le Saint-Laurent) se répercute de façon dramatique sur l'économie québécoise. Ces tensions se traduisent notamment par l'hémorragie de plus en plus massive de population, à compter des années 1830 , vers les villes et les industries de la Nouvelle-Angleterre ${ }^{72}$.

Le régime de fonctionnement du capitalisme commercial a bien démarré en début de siècle. Mais, au Québec, toute une série de «frictions» empêchent le marché d'accomplir pleinement son rôle intégrateur: la disparition des préférences tarifaires réduit la valeur de la colonie comme intermédiaire en même temps que l'échec des efforts pour créer un espace économique commun avec les États-Unis a d'abord limité l'espace économique que Montréal peut polariser; ensuite, l'État, tombé en impasse, n'a pas joué le rôle de support qu'il a assumé ailleurs; enfin, l'hypothèque géotechnique qui a toujours pesé sur ce coin de l'Amérique du Nord maltraité par le climat, n'est pas éliminée par le marché. Toutefois, la commercialisation moins rapide du Québec par rapport à ses voisins du Sud ne signifie pas qu'il n'y a pas pénétration des forces du marché dans tous les aspects de la vie quotidienne. Et si Montréal perd la course contre New York, cette ville s'avère une zone ferme de dynamisation d'un espace commercial encore très éten$\mathrm{du}^{73}$.

L'économie du Québec, au milieu du XIXe siècle, constitue donc une économie de capitalisme commercial qui est bien accrochée au marché atlantique et aboutée au continent nord-américain par les flux de biens, de capitaux, de personnes et d'informations. C'est une économie commercialisée, réglée par les mouvements des prix, avec une architecture institutionnelle à l'avenant et une socioéconomie confrontée à un niveau croissant de concurrence. Mais dans le soubassement de cette économie mercantile, des forces nouvelles font vieillir très rapidement les conflits autour de la détermination des prix. L'industrialisation est à s'implanter.

72 J. McCallum, op. cit., chap. V; G. Paquet et W.R. Smith, op.cit., section 4. On a estimé les flux migratoires vers les États-Unis à plus de 190000 pour la période 1831-1851. Ce régime se serait maintenu au même rythme jusqu'à l'explosion de l'émigration dans le dernier tiers du XIXe siècle.

73 On peut le constater dans G.J.J. Tulchinsky, The River Barons: Montreal Businessmen and the Growth of Industry and Transportation, 1837-1853 (Toronto, 1976). Pour un exemple intéressant des types nouveaux de bourgeois québécois, voir B. Young, GeorgeÉtienne Cartier: Montreal Bourgeois (Montréal, 1981). À. Faucher a étudié la situation privilégiée de l'Ontario dans cette dynamique de développement au coeur du XIXe siècle dans Québec en Amérique au XIXe siècle, chap. VI-VII. 


\section{f) Une troisième grande discontinuité}

Dans la mesure même où s'affirme le marché, comme mécanisme fondamental de coordination des activités au Québec au XIXe siècle, et où s'estompent et disparaissent les arrangements institutionnels mercantilistes ou traditionnels, le Québec se trouve en même temps disloqué et unifié: disloqué puisque ses divers secteurs et régions vont battre à des rythmes différents, définis souvent par des prix déterminés ailleurs, et qui ne sont pas nécessairement harmonisés; unifié également puisque le marché a balayé sur son passage tout un appareil d'organisation socio-économique qui décourageait ou empêchait la mobilité.

L'extension du marché à travers tout le tissu social québécois fait plus qu'instituer un nouveau mécanisme de coordination: parallèlement au procès de dislocation et d'unification par les prix, le marché s'attaque à la substance même de l'organisation sociale. C'est un procès de décomposition plus lent, mais qui transformera encore plus profondément la socio-économie.

Le procès d'industrialisation ne s'accomplit pas brusquement. On peut en déceler quelques signes déjà au XVIIIe siècle (S.B. Ryerson: 08.14), mais il s'agit là d'amorces peu importantes au total. Des percées plus significatives se manifestent dans les villes du Québec dès le premier tiers du XIXe siècle: dans la chaussure, par exemple, où le fractionnement des tâches et le commencement d'un certain émiettement du travail résultent de la pression des forces du marché qui poussent vers l'accroissement de la productivité et une réduction des prix. La technologie n'a pas encore vraiment changé par rapport à ce qu'elle était au temps de la NouvelleFrance. C'est l'organisation du travail autour de cette technologie qui a évolué (J. Burgess: 12.3ss) ${ }^{74}$.

Ce n'est qu'avec les années 1840 et l'ouverture du canal Lachine en 1848 qu'on peut vraiment parler des débuts de l'industrialisation au Québec. La zone du canal Lachine deviendra en quelque sorte le coeur industriel du Canada (P.-A. Linteau: 12.13). Mais l'explosion qui provoquera la discontinuité dans tout le procès social au Québec ne surviendra que dans le dernier tiers du XIXe siècle: montée de la fabrique, aboutissement logique du procès d'éclatement des tâches, remplacement par des machines des ouvriers non spécialisés effectuant des tâches routinières, bref passage de la manufacture à la machino-facture.

${ }^{74}$ J. Burgess, «L'industrie de la chaussure à Montréal: 1840-1870 - Le passage de l'artisanat à la fabrique», RHAF, 31,2 (septembre 1977): 187-210. 
Avec la fabrique, tout l'appareil d'organisation socioéconomique est transformé: la clé de l'architecture socioéconomique ne réside plus dans le marchandage autour du prix, mais dans la définition d'un prix particulier qui est au centre de tout l'appareil, le prix du travail, le salaire. Non seulement la fabrique bouleverse les conditions de travail et consomme l'expropriation des compétences des artisans désormais rendus inutiles, mais elle modifie toute la trame de la vie québécoise (F. Harvey: 14.16ss).

Dans le monde de la machino-facture, les ouvriers sont autant de pièces interchangeables - c'est la dégradation de la qualification du travail. C'est aussi l'urbanisation à proportion même du besoin de cette masse ouvrière indéfinie dans les lieux particuliers que sont les villes industrielles en train de croître. La mécanisation et l'urbanisation accrue imposent un genre de vie très différent à la population québécoise et donnent lieu à un contre-mouvement de syndicalisation: dès les années 1880 , on peut parler d'un mouvement ouvrier organisé (J. Rouillard: 14.17) ${ }^{75}$.

Cette période enregistre également les effets d'une transition démographique: le taux de mortalité baisse et, avec un peu de retard, le taux de natalité aussi. Dans l'ensemble, la population s'accroît de façon dramatique (H. Charbonneau: 14.8). Et malgré la multiplication des machino-factures et l'accélération de l'industrialisation (l'enquête Blackeby révèle qu'entre 1878 et 1884, plus de 25000 emplois ont été créés dans les fabriques du Canada central) ${ }^{76}$, le trop-plein des hommes nourrit une émigration extraordinaire de Québécois vers les États-Unis: entre 1880 et 1900 , on dénombre au moins 290000 départs (Y. Lavoie: 15.19) ${ }^{77}$.

À ces changements importants dans le sous-procès démographique et celui de la production et de l'échange, correspondent des modifications parallèles dans le sous-procès financier: c'est la transformation des institutions financières en outils d'accumulation de capital. En sept ans, entre 1868 et 1874, surgissent 20 nouvelles banques. Les compagnies d'assurance bénéficient d'une croissance très forte et se transforment d'ailleurs, passant de socié-

\footnotetext{
J. Rouillard, Les syndicats nationaux au Québec de 1900 à 1930 (Québec, 1979), chap. I et II; E. Forsey, The Canadian Labour Movement, 1812-1902 (Ottawa, 1974).

${ }_{76}$ Report Relative to Manufacturing Industries in Existence in Canada, Sessional Papers no 37, 1885. Ce document revêt un caractère un peu apologétique: il célèbre la «Politique nationale», la stratégie de développement économique de l'État canadien et ses effets positifs sur l'emploi. Au Québec, selon les recensements, le nombre de personnes employées dans le secteur industriel passe de 26000 en 1851 à 65000 en 1871 et à 93000 en 1891.

Y. Lavoie, «Les mouvements migratoires des Canadiens entre leur pays et les États-Unis au XIXe et au XXe siècles: étude quantitative», in H. Charbonneau, éd., $L a$ population du Québec: études rétrospectives (Montréal, 1973), 78.
} 
tés d'entraide mutuelle au statut d'instruments d'accumulation de capital proprement dits ${ }^{78}$.

Dans la mesure même où l'accumulation du capital requis pour la construction de fabriques et la mise en place de moyens de production à plus forte intensité de capital réclament un système financier plus stable et des formes d'entreprise nouvelles, le cadre légal s'adapte et l'État joue un rôle d'appui, voire de catalyseur pour le système économique en pleine mutation dans le dernier tiers du XIXe siècle, au moment même où une grande récession économique affecte l'économie mondiale ${ }^{79}$.

En fait, l'industrialisation de la fin du XIXe siècle constitue l'aboutissement d'une logique du marché qui pénètre partout: non seulement ce dernier règle-t-il le jeu des transferts de marchandises, mais il dissout finalement tous les rapports sociaux antérieurs qui deviennent coordonnés par lui et par une sorte de concurrence effrénée. C'est tout au moins ce qui sourdrait sans l'émergence d'un contre-mouvement qui accompagne, bien que faiblement au début, l'enclenchement de la mutation. En un sens, tout le régime de fonctionnement de la période de capitalisme industriel, après la troisième discontinuité, sera caractérisé par un faisceau de contremouvements s'opposant à la logique corrosive de la mécanisation et du marché et construisant un régime d'institutions privées, publiques ou communautaires pour la contrer ${ }^{80}$.

\section{g) Un régime de capitalisme industriel}

Si la machino-facture et la position centrale du salaire comme le prix-clé dans l'organisation économique engendrent un contremouvement rapide dans le recours accru des ouvriers à l'action

78 D. Bond et R.A. Shearer, The Economics of the Canadian Financial System, (Scarborough, 1972), chap. XII.

${ }_{79}$ Pour un coup d'oeil général sur les grandes lignes de l'évolution de la socio-économie canadienne et québécoise au cours de cette période, pour laquelle des chiffres plus fidèles sont disponibles, voir O.J. Firestone, Canada's Economic Development, 1867-1953 (London, 1958), et les articles de A. Gosselin et A. Dubuc in R. Comeau, éd., Économie québécoise (Montréal, 1969).

${ }_{80}$ Tant le mouvement de cartellisation parmi les entreprises et d'intervention étatique par divers gouvernements que la montée des coopératives et des syndicats ouvriers peuvent être considérés comme la mise en place de moyens pour contrer ou limiter l'action du marché et du mécanisme des prix. On a donc pu arguer qu'après la troisième grande discontinuité s'est opérée une sorte de retour en arrière, les règles du jeu dans le nouveau régime de fonctionnement rappelant à certains égards la réglementation en régime mercantiliste. De même, comme dans l' «ancien régime», les problèmes de répartition de revenu prennent un peu plus d'importance qu'ils n'en avaient durant les belles années du capitalisme commercial. 
collective, à la grève et à la syndicalisation, la concurrence énorme au sein de ce régime de capitalisme industriel et la nécessité d'une accumulation du capital de plus en plus rapide suscitent d'autres formes de réaction et d'ajustement du régime de fonctionnement de la socio-économie de façon à limiter les dégâts d'une concurrence trop forte. Dès les années 1890 , les entreprises commencent à réagir à la concurrence: fusions d'entreprises, plus grande concentration du pouvoir économique. Cette évolution vers la concentration conduit d'ailleurs à la disparition, ou tout au moins à la marginalisation, de la classe d'entrepreneurs canadiens-français qui avait émergé dans la seconde moitié du XIXe siècle (P.-A. Linteau: $17.18 \mathrm{ss})$.

Le Québec a donc participé à cette première vague d'industrialisation de la fin du XIXe siècle, comme le démontrent bien les études sur Montréal et sa région à cette époque. Mais le mouvement de concentration du pouvoir économique, non seulement au Canada mais au niveau du continent, donne un deuxième souffle, si l'on peut dire, à l'industrialisation du Québec au XXe siècle. Il s'agit cette fois d'une industrialisation engendrée par l'influx de capitaux américains au Canada en vue notamment de l'exploitation des ressources naturelles du Bouclier laurentien. L'interaction entre les ressources naturelles qu'on veut exploiter et l'importance croissante de l'hydro-électricité accentuera la concentration de l'industrie légère au Québec. L'industrie lourde, qui dépend davantage de l'acier et du charbon que l'Ontario peut se procurer à meilleur compte, se localisera dans cette province ( $\mathrm{R}$. Armstrong: $21.12)^{81}$.

L'histoire de la socio-économie québécoise dans les deux premiers tiers du XXe siècle, c'est celle d'un capitalisme industriel qui cherche par toute une série d'institutions à régler un régime de fonctionnement qui a des ratés de plus en plus sérieux. Ainsi l'État assume un rôle nouveau, et de plus en plus ambitieux. Déjà, il est intervenu dans les années 1870 avec la loi des banques et les politiques nationales; mais à l'occasion de la Première Guerre mondiale, puis de la crise des années 1930 et des rêves de welfare state après la Seconde Guerre mondiale, il se gonfle à un rythme effréné non seulement par ses revenus en pourcentage du PNB (produit national brut), mais aussi dans une jungle de cadres réglementaires ${ }^{82}$.

81 Sur l'industrialisation du Québec après la troisième grande discontinuité, voir $\mathrm{J}$. H. Dales, «Fuel, Power and Industrial Development in Central Canada», American Economic Review (mai 1953); R. Durocher et P.-A. Linteau, éd., Le «retard» du Québec et l'infériorité économique des Canadiens français (Montréal, 1971).

${ }_{82}$ Pour une vue globale de cette dérive de la socio-économie québécoise, voir $\mathbf{R}$. Durocher, P.-A. Linteau et J.-C. Robert, Histoire du Québec contemporain, chap. IV-VII, XVIII-XXIV. 
Tout au long de cette période, le Québec continue à s'urbaniser, à se syndiquer, et à se doter aussi d'institutions communautaires pour échapper à la discipline du marché: montée des coopératives, développement d'un syndicalisme qui fera de plus en plus passer les décisions quant au niveau des salaires depuis les impératifs du marché à ceux des négociations collectives. Mais la socio-économie ne maintient pas une vitesse de croisière soutenue dans cette évolution. Par exemple, la Seconde Guerre mondiale affermit une volonté de rattrapage au Québec. D’ailleurs, la réalité démographique change dramatiquement: c'est l'urbanisation déchaînée des années 1940, mais aussi les effets extraordinaires de retombée ni voulus ni prévus du «baby boom» de l'après-guerre. Déjà mieux armé idéologiquement depuis la grande crise pour jouer un rôle de mécanisme régulateur de l'économie, l'État prend en charge des rôles nouveaux de régulation sociale et grossit ses budgets de façon spectaculaire dans les domaines de la santé et de l'éducation, face à cet accroissement de la population ${ }^{83}$.

On voit aussi se consolider tout le sous-système financier avec un éventail de nouvelles institutions qui viennent combler les lacunes dans l'appareil de mobilisation et de distribution de l'épargne. Toutefois, malgré l'ajout de services nouveaux et d'instruments financiers particuliers dans les années 1950, l'armature du système financier, avec la Banque du Canada pour pivot, ne change guère en profondeur (H.B. Neatby: 20.10) ${ }^{84}$.

Le Québec éprouve une double industrialisation (celle qui se greffe aux ressources naturelles de la périphérie et la montée de l'industrie légère dans les villes) comme régime de croissance extensive: c'est l'addition de capital étranger et de main-d'oeuvre en provenance des campagnes qui nourrit le procès de développement. La croissance s'essouffle dans les années 1930; mais elle rebondit de façon presque explosive avec la Seconde Guerre mon-

83 J. Kettle, The Big Generation (Toronto, 1980). Pour différentes perspectives sur ces changements, voir J.-C. Falardeau, éd., Essais sur le Québec contemporain (Québec, 1953); A. Faucher et G. Paquet, «L'expérience économique du Québec et la Confédération", in Revue d'études canadiennes (novembre 1966): 16-30; P. Desbiens, "Perspectives sur l'État québécois», in P. Fournier, Le capitalisme au Québec (Montréal, 1978), 109-133.

${ }_{84}$ On ne décèle pas de transformation fondamentale des institutions financières. Ce n'est que lentement que la Banque du Canada qui, dans les années 1930, était contrôlée par les banquiers plutôt que par le gouvernement, acquiert son indépendance. L'importance relative des banques décroîtra: de $75 \%$ des actifs des intermédiaires financiers qu'elles détenaient en 1870, la part des banques chute à $45 \%$ en 1930 et se maintiendra à ce niveau jusque dans les années 1970. Toutefois, elles demeurent au centre de l'appareil économicofinancier. Et tout au cours de l'entre-deux-guerres, on voit un mouvement de concentration des banques à charte, une sorte de «molécularisation» du système bancaire sans que les règles du jeu ne soient modifiées en profondeur. Voir G. Piédalue et J. Niosi in cahier 22, passim. 
diale, qui hausse le taux de participation à la main-d'oeuvre des ressources humaines disponibles, et dans l'après-guerre, avec l'envahissement de la socio-économie québécoise par les capitaux américains sur une nouvelle échelle.

Dans les années 1940-1950-1960, revenu et produit domestique grimpent en parallèle: la croissance du premier est assuré, le cas échéant, par l'action de l'État qui, depuis les années 1940, s'est engagé à maintenir le niveau du revenu et de l'emploi (livre blanc sur le revenu et l'emploi de 1946); celle du second est stimulée par la réduction des barrières douanières (qui assure l'extension des marchés) ainsi que par l'injection de capital étranger et l'urbanisation des Québécois qui, en passant à la ville, augmentent leur productivité. À mesure que sailliront des signes de défaillance de l'entrepreneurship privé dans les années 1960, apparaîtra un entrepreneurship public pour animer cette croissance extensive ${ }^{85}$.

Si le capitalisme industriel semble changer de face, le régime de fonctionnement, quant à lui, varie très peu. Les études d'André Raynauld, dans les années 1960, démontrent que la vague de concentration des entreprises dans l'après-guerre n'a fait que confirmer les effets de la première vague de fusion du début du siècle qui avait balayé l'entrepreneurship autochtone naissant: dans leur sillage, ces vagues laissent un dualisme de pouvoirs économiques au Québec qu'on peut repérer même dans l'espace - anglo-américain à Montréal, canadien-français dans les régions excentriques ${ }^{86}$.

Avec les années 1960, la croissance extensive ralentit, au Québec comme ailleurs. L'abaissement des barrières douanières et l'urbanisation ont fini de produire leurs effets. On glisse lentement vers un régime de croissance intensive. Or, celle-ci dépend complètement de l'accroissement de la productivité, i.e. du «know how», du design, de l'innovation technique et de la recherche. Elle s'avère donc plus difficile et plus lente, surtout dans une socioéconomie dont la progression extensive a été orchestrée de l'extérieur. Mais les droits sociaux nouveaux acquis dans l'après-guerre - tels les droits au travail, à l'éducation, à la santé, au maintien du revenu, à la participation à l'enrichissement collectif - commencent à être revendiqués avec force. Les gouvernements les consentent ou les sanctionnent avec une générosité un peu euphorique au moment même où la productivité commence à progresser plus lentement et où, à cause de la reconnaissance de ces droits, les

\footnotetext{
85 P. Desbiens, op.cit., 122-155; G. Paquet, «Un État empêtré dans une société temporaire», in C. Ryan, éd., Un Québec qui se fait (Montréal, 1971), 123-128.

86 A. Raynauld, La propriété des entreprises du Québec (Ottawa, mai 1967).
} 
contre-mouvements qui voulaient limiter la pénétration du marché, réussissent à aménager dans le tissu social des zones de plus en plus vastes qui échappent au marché.

Ces facteurs débouchent sur une socio-économie québécoise plus stable, mais plus fragmentée, moins malléable et plus lente à s'ajuster. De la sorte, le régime de fonctionnement institué au XIXe siècle pour promouvoir la croissance économique sous la seule contrainte que les coûts sociaux n'en soient pas trop exorbitants, en arrive à se transformer, dans le "second XXe siècle», en un régime de fonctionnement qui semble plutôt institué pour minimiser les risques individuels et collectifs et qui accorde de plus en plus de place à des mécanismes de coordination des activités économiques autres que le marché ${ }^{87}$.

\section{h) Une quatrième grande discontinuité}

La vision qui sous-tend les analyses du capitalisme industriel, met l'accent sur la production de biens matériels et sur le rôle central du travail dans ce procès. Le marché est l'institution tout à fait ajustée aux transferts de marchandises qui constituent l'aboutissement de ce procès. Et il en ressort un déterminisme technicogéographique plus ou moins contraignant qui pèse sur la localisation des activités économiques dans l'espace. En fait, les explications des contours de l'industrialisation au Québec jusque dans les années 1950, reposent surtout sur ces facteurs ${ }^{88}$.

Mais il s'est produit dans l'après-guerre une transformation fondamentale des socio-économies des pays avancés que l'on n'a pas suffisamment notée, une mutation à laquelle le Québec a pleinement participé. Nous pourrions l'identifier comme un procès de désindustrialisation, i.e. un déclin de l'importance relative du secteur industriel de la production de biens matériels dans la somme des activités économiques du Québec. Cette désindustrialisation s'est traduite par une dématérialisation et, par voie de conséquence, par une «déterritorialisation» de l'activité économique: celle-ci est devenue beaucoup moins tributaire des conditions géo-

87 Pour un coup d'oeil sur l'évolution de la socio-économie québécoise et canadienne vers une unité économique plus fragmentée mais plus stable à cause même de cette fragmentation («multi-stable») en secteurs et zones réglementés sujets à des règles du jeu différentes et en interaction par le truchement de canaux spécifiques, voir G. Paquet, «The Regulatory Process and Economic Performance», in G.B. Doern, ed., The Regulatory Process in Canada (Toronto, 1978); idem, «Federalism as Social Technology», in J. Evans, ed., Options (Toronto, 1977).

88 J.H. Dales, op.cit. 
techniques du milieu physique et relève beaucoup plus de facteurs liés à la technologie sociale ${ }^{89}$.

On a souvent abordé ce type de changement en faisant référence à la société post-industrielle ou à la société de services. Sans être fausse, cette approche n'éclaire pas l'aspect essentiel de cette transsubstantiation de la socio-économie. De la même façon, on a insisté sur la montée de la production étatique et même parlé d'un capitalisme d'État sans se rendre compte qu'il s'agissait souvent d'un épiphénomène qui découle justement du fait que la socioéconomie en est venue lentement à produire de moins en moins de biens matériels et de plus en plus d'information ${ }^{90}$.

La notion d'information est complexe; mais il est possible de définir clairement et de mesurer la portion de l'économie qui est affectée à la manipulation de symboles. Des études ont montré qu'au début des années 1970, la proportion des emplois qu'on pouvait classifier dans le secteur «information» frisait $40 \%$ au Canada et dépassait 50\% aux États-Unis. Il s'agit évidemment d'évaluations grossières. Mais elles mettent en évidence l'importance croissante de ce secteur dynamisant dans la structure de nos socio-économies ${ }^{91}$.

89 Déjà, certaines analyses ont sondé cette mutation à la fin des années 1960 (v.g. F. Perroux, Industrie et création collective, tome II (Paris, 1970): chap. II); mais la dichotomie se trouve au centre des débats au milieu des années 1970 (J. Attali, La parole et l'outil, Paris, 1975). Une étude majeure du problème en contexte américain existait déjà en 1974 (M.U. Porat, The Information Economy, miméo, Stanford, 1974) et le groupe GAMMA de Montréal a produit un rapport important sur les réalités canadienne et québécoise en 1979(K. Valaskakis, The Information Society: The Issue and the Choices, Montréal, 1979). Pour un examen des implications de cette mutation dans la socio-économie sur les opérations des entreprises plurinationales et sur le sous-procès de travail, voir $\mathrm{S}$. W. Langdon et G. Paquet, The Multinational Enterprise and the Labour Process, O.E.C.D. (Paris, 1976). Le hiatus dans l'expérience de l'économie du Québec est noté par P. Fréchette, R. Jouandet-Bernadat et J.-P. Vézina, L'économie du Québec (Montréal, 1975), chap. IV, mais est expliqué autrement.

90 Pour un examen de la désindustrialisation et de ses conséquences pour le développement de l'économie non marchande comme mécanisme de coordination, voir G. Paquet, "L'économie non marchande dans l'économie de marchés", Revue d'économie politique (juillet-août 1977).

91 M.U. Porat, The Information Economy (Washington, 1977) et K. Valaskakis, op.cit., 16. Ces données tentent seulement d'identifier les activités de production de connaissance et de transfert d'information dans la socio-économie en spécifiant les transactions d'information comme telles (design, éducation, recherche, communication, etc.) et tout le support informationnel des bureaucraties publiques et privées qui appuie à la fois les 
On n'a pas encore procédé à une bonne analyse de la dématérialisation de la vie économique québécoise, et les données pour le faire n'existent pas. Mais il est clair que cette transformation s'est répercutée sur tous les domaines: elle a été accélérée par la turbulence et la complexité accrues des procès économiques à mesure que l'économie s'est ouverte dans l'après-guerre; elle a aussi été catalysée par le «boom» démographique (l'injection dans la société québécoise d'une cohorte de deux millions de personnes nées entre 1951 et 1966) et la demande de services qui s'ensuivit ${ }^{92}$. Mais elle a également agi sur ces réalités. Les phénomènes sociopolitiques comme la Révolution tranquille, la montée de l'État, les nouvelles formes de coordination de l'activité économique, la possibilité même de planifier davantage et de redéployer les ressources humaines et financières au nom de nouveaux nationalismes économiques - tout cela devient possible à cause de la dématérialisation de la vie économique (A. Raynauld: 25.17; A. Rotstein: 25.19ss).

Cette dernière transition vers une économie de l'information, une économie qui se désindustrialise et devient immatérielle, certains croient en dépister les signes clairs dès les années 1950. Mais elle s'accomplit dans les années 1960-1970 et elle n'est pas encore achevée. (Cahier 25. Passim).

\footnotetext{
transactions d'information et les autres. Il s'agit donc d'un effort pour distinguer ceux dont le travail consiste à manipuler des symboles de ceux qui dépensent de l'énergie physique. Cette distinction entre information et énergie est centrale: la dernière discontinuité résulte d'un changement dramatique dans le ratio information/énergie dans l'économie. Une autre analyse, davantage micro-économique, explique la dichotomie comme suit: "There are two traditional divisions of the economy, by industry and by employment. Beyond the categorization by product of the industry and personnel employed, there is an undeveloped but extremely useful categorization which depends largely on the nature of the job; this is the separation by information and energy processes. Shipping from the docks of Manhattan is fundamentally an energy process, whereby a certain number of tons of payload are physically moved from New York to London, for example. The ships that carry them are a particular kind of energy machine, as are airplanes and taxicabs. But the process of preparing the bills of lading, of controlling the destination of the cargo and the navigation of the ship are information processes in that no physicals mass is transported.» (J. McLaughlin, Information Technology and Survival of the Firm (Homewood, Ill., 1966), 12). McLaughlin procède alors à une classification des procès informationnels en 4 groupes (14ss): 1 - ceux qui sont entièrement informationnels (éducation, banque, finance, assurances, etc.); 2 - le contrôle de la distribution des biens (toute la fonction de mise en marché); 3 - le contrôle des opérations (systèmes de gestion, informatique, etc.); 4 - les procès informationnels imputés (toutes sortes d'occupations qui étaient autrefois physiquement épuisantes et qui ont été transformées en manipulation de symboles).
}

92 J. Kettle, op.cit. 


\section{i) La socio-économie d'information}

Il peut paraître hasardeux de vouloir caractériser un régime de fonctionnement encore en train de prendre. À cause de l'immédiateté de l'objet de l'enquête, l'histoire (si c'en est encore) doit se rapprocher ici davantage de la conjecture. Pourtant, une évocation fort sommaire et par définition incomplète des lignes de force de ce régime peut quand même permettre une première saisie de cette dynamique nouvelle ${ }^{93}$.

Dans la socio-économie d'information, le secteur non marchand prend une place de plus en plus grande parce que le marché n'est pas un véhicule efficace pour le transfert de l'information ${ }^{94}$. La turbulence accrue engendre un procès d'oligopolisation croissante et la firme plurinationale est en fait une institution particulièrement apte à tirer profit d'économies d'informations importantes dans un monde instable où ces économies comptent de plus en plus. Le passage de l'internationalisation des opérations à une véritable internationalisation du procès de production par les plurinationales survient avec le passage à la socio-économie d'information $^{95}$.

Dans ce contexte, les prix sont déterminés non par des marchés libres, mais par des institutions chargées de le faire (offices de mise en marché, régies, commissions gouvernementales). Même les grandes entreprises en arrivent à définir leurs prix beaucoup plus en rapport avec leurs besoins de trésorerie (dans un monde d'autofinancement des grandes firmes) qu'en rapport avec la concurrence sur le marché libre ${ }^{96}$.

Une portion de plus en plus grande de la population active oeuvre dans le secteur dit «tertiaire» et en particulier dans le secteur public. Les contraintes géo-techniques perdant de leur poids, on voit surgir l'importance de la technologie sociale et la désagré-

93 Pour fixer les idées, disons qu'une socio-économie d'information existe dans les définitions de Porat et de Valaskakis lorsque $50 \%$ du produit national brut appartient au secteur de l'information, i.e. au secteur de la manipulation de symboles plutôt qu'à la manipulation de biens matériels. On peut penser qu'au Québec, on en est à peu près là en 1982. Pour un examen des implications pour tous les sous-procès de cette informatisation de la société, voir S. Nora et A. Minc, L'informatisation de la société (Paris, 1978) et les études multiples du groupe GAMMA que résume le rapport de K. Valaskakis cité plus haut. 94 D.M. Lamberton, ed., Economics of Information and Knowledge (Hardmondsworth, 1971).

95 S.W. Langdon et G. Paquet, op.cit.

96 Pour un examen critique des contours de cette socio-économie en train de s'instituer, voir W.D. Slawson, The New Inflation (Princeton, 1981). Il ne s'agit pas d'annoncer la mort du marché libre qui subsiste évidemment dans nombre de secteurs. Mais le prix est de plus en plus défini non seulement dans les secteurs de pointe, mais même dans la périphérie, par des institutions qui prennent leurs distances par rapport au marché libre et donc par rapport au procès de concurrence qui sous-tend le marché libre. 
gation, la désintégration des procès physiques de production: c'est le monde de la sous-traitance et donc la croissance, à côté d'un secteur informationnel fort, où les emplois sont bien rémunérés et stables, d'un secteur de vacataires, de pigistes dont les contrats sont à durée limitée, la stabilité d'emploi, faible, et la rémunération, aléatoire ${ }^{97}$.

En réaction à ce contexte turbulent s'impose une sorte de besoin de reconquête de la certitude dans toutes les directions: on élimine le marché libre du monde du travail; alors que la population commence à vieillir, c'est moins la productivité qu'on recherche que l'indexation des salaires et le droit à l'immobilité; la réduction du protectionnisme formel (droits de douanes, barrières à l'entrée des banques étrangères, etc.), suite aux négociations internationales, s'accompagne d'un protectionnisme déguisé et croissant, d'un ensemble de politiques destinées à «protéger» la population différemment, mais encore plus fermement.

Dans une socio-économie où les droits sociaux ont été établis au cours d'une période de croissance économique rapide, les ajustements et le redéploiement deviennent difficiles en période de transition, surtout que ces ajustements se traduisent par une expropriation de certains droits qu'on croyait acquis. Le grand jeu québécois est en train de chercher ses règles nouvelles, lentement, car pour le moment, malgré le déclin de la productivité, la chute des investissements, le chômage élevé et l'inflation institutionnalisée, personne n'en est arrivé à croire qu'il faudra abandonner en fait certains de ces droits acquis dans l'après-guerre.

En attendant, l'internationalisation de la production provoque chaque semaine des départs d'entreprises pour les zones de bas salaires des pays-ateliers du Tiers-Monde et le raffermissement de l'équilibre conflictuel entre régions, secteurs et groupes. On est loin du consensus: c'est l'empoigne généralisée autour du pot-aubeurre et le règne de l'idéologie puisque la discipline du marché et des contraintes physiques a disparu. Dans le marchandage, chacun peut encore espérer obliger les autres à s'adapter. Reste à savoir combien de temps il faudra avant que la détérioration de la situation ne force les Québécois à prendre les moyens pour que leur socio-économie s'institue différemment. Pour certains, c'est un

97 A. Lipietz, «Redéploiement industriel: le legs du libéralisme», Le Débat (novembre 1981): 39-49. C'est là une formule pour réduire les coûts et affronter la concurrence, mais par une dégradation de la qualité de vie d'une portion de la main-d'oeuvre. On voit aussi en parallèle la recherche (à la fois à l'intérieur et à l'extérieur du Québec) de zones de bas salaires où on veut exporter les usines. 
procès de changement sociétal lent dont il ne faut pas attendre la cristallisation avant le milieu des années $1980^{98}$.

Quant aux contours des institutions de cette socio-économie d'information, on peut tout au plus spéculer à leur sujet. Mais il est certain qu'on verra le passage à une socio-économie éclatée, fractionnée, où la petite organisation l'emportera en productivité sur la grande, et où les conflits opposeront bien moins capital et travail que des «groupes mobiles et innombrables conditionnés par la diversité de leur appartenance et de leurs projets»» ${ }^{99}$.

\section{Le pouvoir heuristique de cette problématique}

L'élaboration d'un instrument de prospection à une telle échelle, d'une sorte de macroscope qui découpe aussi simplement une réalité fort complexe, comporte évidemment des risques nombreux. On pourra reprocher à cet arrangement préalable des objets de l'enquête de ne pas être entièrement satisfaisant, à notre méthode de prospection, de manquer de systématisation, à notre hypothèse, de ne pas être vraiment concluante.

Nous ne pourrions nous objecter à aucun de ces reproches. Pourtant, ils portent à faux. En effet, notre premier objectif, comme nous l'avons souligné, est de composer un agencement préliminaire des informations connues, un cadre général pour mieux organiser le dossier documentaire. En ce sens, cette première esquisse a une vocation instrumentale: elle nous a paru utile pour examiner l'expérience historique de la socio-économie québécoise à travers plusieurs mutations et même pour éclairer nos analyses de cette réalité dans les années 1980 . C'est là un mérite que les schémas alternatifs n'ont pas démontré.

En outre, ce cheminement guidé peut aider à contester les explications univoques des changements dans la socio-économie québécoise qui sont parfois avancées dans l'historiographie. Le cadre d'un article ne pouvait nous permettre d'examiner et de met-

98 Pour un coup de sonde du côté des ajustements de la socio-économie dans les années 1980 à partir d'une analyse des six sous-procès de base, voir G. Paquet «Putting it All Together: A Political Economy Perspective of the Early 1980's", in C.A. Barrett, ed., Key Economic and Social Issues of the Early 1980's (Ottawa, 1980).

99 S. Nora et A. Minc, op.cit., 114. Le rapport Nora-Minc développe à la fois les futuribles et les probables pour cette socio-économie qui est en train de prendre. Groupes sociaux, pouvoirs, finances, structures de production, administrations, sont autant de domaines qu'explore ce rapport. On a aussi déjà construit en contexte canadien des scénarios plausibles pour les années 1980 (K. Valaskakis, op.cit., chap. IV). Notons que cette dernière grande discontinuité a un fondement technologique certain, mais qu'elle annonce une société qui tant dans sa structure que dans la théorie qu'elle se donne d'elle-même, n'est pas nécessairement nouvelle. Certains ont pu parler de la nécessité de socialiser l'information, d'autres, d'un nouveau mercantilisme. 
tre en valeur tous les différents éléments qui composent le tableau dynamique de cette socio-économie. Mais notre approche suggère tout au moins pour chaque discontinuité et chaque régime de fonctionnement une série de questions importantes qu'aucune analyse sérieuse ne peut laisser entièrement de côté. Elle implique clairement que ceux qui veulent faire porter tout leur explicandum sur l'un ou l'autre des sous-procès, devront pouvoir expliquer clairement comment leur interprétation éclaire les évolutions dans les autres sous-procès. À l'inverse, des changements dans un seul sous-procès, sans répercussions sur les autres, pourraient bien n'être que des réajustements internes, des réaménagements de façade, tout au plus des étapes, mais non des discontinuités majeures.

Notre problématique devrait donc permettre de mieux sérier les problèmes et de situer les controverses en perspective. Par exemple, la notion de la transition du féodalisme au capitalisme n'est pas inutile en soi, mais elle n'a pas accouché de schémas explicatifs bien fermes et bien enracinés dans des données empiriques pertinentes au contexte québécois et canadien. L'approche par les discontinuités et les régimes de fonctionnement permet de désagréger un procès majeur de l'histoire occidentale qui ne s'est probablement jamais accompli entièrement en territoire nordaméricain, en une série de tronçons, de «respirations» sociétales toujours complexes, certes, mais susceptibles d'analyses liant les six sous-procès fondamentaux de la socio-économie.

Même les controverses peuvent changer de sens et de direction, une fois insérées dans une trame plus précise. Les lacunes dans nos connaissances ressortent encore plus clairement aussi et peuvent stimuler des explorations dans plusieurs sous-procès trop longtemps ignorés. Il n'est pas question ici d'aligner toutes les controverses majeures de l'historiographie ou de repérer les «trous» dans la constellation des faits connus. Qu'on nous permette seulement quelques exemples. L'ancienne «rupture» majeure sous Talon a-t-elle vraiment l'importance de la discontinuité du début du XVIIIe siècle? N'est-ce pas comparer la quasi-naissance d'une colonie à un moment (le plus mal connu, peut-être, dans l'historiographie) d'évolution de plus en plus complexe et rapide de la société canadienne? La controverse de la «rupture» de la Conquête relancée encore tout récemment perd son sens dans notre schéma, qui retient pourtant l'importance du phénomène capital de la substitution de métropole, d'administrateurs et de grands marchands. La seconde discontinuité rend compte bien davantage que la Conquête ou une crise agricole de la modernisation du Bas-Canada et des conséquences de la pénétration du marché dans la société, de façon à mieux expliquer les évolutions ultérieures. Pourra-t-on. se con- 
tenter d'imputer le retard industriel du Québec - et, dans certains cas, du Canada - aux seules frictions présumées du capital marchand et du capital industriel, ou encore aux seules mentalités (avec ou sans la médiation de la technologie)? Le concept de capitalisme d'État recouvre-t-il bien la réalité du «second XXe siècle»? Le nationalisme et les conflits politiques peuvent-ils être compris en faisant abstraction des nombreux sous-procès de la socioéconomie? Dans tous ces cas comme en tant d'autres, en effet, l'analyse achoppe si elle ne tente pas de suivre les ramifications et les interactions entre sous-procès. À la limite, d'autres schémas peuvent déplacer les discontinuités, en montrer d'autres ou les expliquer autrement. Mais pour ce faire de façon convainquante, ils devront eux aussi dénouer de façon explicite l'écheveau complexe des forces motrices qui instituent une socio-économie différemment de lieu en lieu et de moment en moment.

\section{Conclusion}

Le danger du travail au macroscope, c'est non seulement le risque d'une simplification outrancière, mais aussi le plaisir d'ordonner trop bien ce qui peut paraître d'une complexité irréductible au premier abord. Pourtant, la timidité de beaucoup d'historiens qui refusent de suggérer même de façon générale les contours de leur vision du monde, est-elle défendable dans une discipline qui se veut une "science sociale»? Il ne nous paraît pas possible d'effectuer quelque travail que ce soit en sciences humaines sans qu'il ne soit entaché d'une manière ou d'une autre des valeurs des chercheurs. Aussi bien se rendre à l'évidence que l'explicitation de ces valeurs demeure encore la meilleure voie d'«objectivation». Aussi avons-nous tenté à quelques reprises, au cours des derniers quinze ans, de faire le point publiquement sur notre vision des choses, sur notre «weltanschauung» qui de toute évidence évolue à proportion que progressent nos travaux en extension et en profondeur.

Nous croyons que le point de départ que nous nous sommes donné dans une perception de la socio-économie comme procès institué décomposable, a un pendant dans une définition de rationalité plus «substantive» et dans une approche plus ample qui permet d'éluder la fixation sur l'échange marchand qui a hanté les travaux néo-institutionnels des dernières années. Nous sommes conscients que les fondements théoriques et les travaux empiriques nécessaires à la vérification de la série d'hypothèses présentées ici, exigeront beaucoup de temps et d'efforts. Notre espoir est que ce propos d'étape provoque tout au moins quelques questions intéressantes qui autrement n'auraient peut-être pas été posées. 